\title{
Parameter estimation for exponential sums by approximate Prony method
}

\author{
Daniel Potts ${ }^{\mathrm{a}}$, Manfred Tasche ${ }^{\mathrm{b}}$ \\ ${ }^{a}$ Chemnitz University of Technology, Department of Mathematics, D-09107 Chemnitz, Germany \\ ${ }^{b}$ University of Rostock, Institute for Mathematics, D-18051 Rostock, Germany
}

\begin{abstract}
The recovery of signal parameters from noisy sampled data is a fundamental problem in digital signal processing. In this paper, we consider the following spectral analysis problem: Let $f$ be a real-valued sum of complex exponentials. Determine all parameters of $f$, i.e., all different frequencies, all coefficients, and the number of exponentials from finitely many equispaced sampled data of $f$. This is a nonlinear inverse problem. In this paper, we present new results on an approximate Prony method (APM) which is based on [1]. In contrast to [1], we apply matrix perturbation theory such that we can describe the properties and the numerical behavior of the APM in detail. The number of sampled data acts as regularization parameter. The first part of APM estimates the frequencies and the second part solves an overdetermined linear Vandermonde-type system in a stable way. We compare the first part of APM also with the known ESPRIT method. The second part is related to the nonequispaced fast Fourier transform (NFFT). Numerical experiments show the performance of our method.
\end{abstract}

Key words: Spectral analysis problem, parameter estimation, exponential sum, nonequispaced fast Fourier transform, digital signal processing, approximate Prony method, matrix perturbation theory, perturbed Hankel matrix, Vandermonde-type matrix, ESPRIT method.

AMS Subject Classifications: 94A12, 42C15, 65T40, 65T50, 65F15, 65F20.

\section{Introduction}

We consider a real-valued sum of complex exponentials of the form

$$
f(x):=\sum_{j=-M}^{M} \rho_{j} \mathrm{e}^{\mathrm{i} \omega_{j} x} \quad(x \in \mathbb{R})
$$

with complex coefficients $\rho_{j} \neq 0$ and real frequencies $\omega_{j}$, where

$$
\omega_{0}=0<\omega_{1}<\ldots<\omega_{M}<\pi \text {. }
$$

For simplicity, we discuss only the case $\rho_{0} \neq 0$. Since $f$ is a real function, we can assume that

$$
\omega_{j}=-\omega_{-j}, \quad \rho_{j}=\bar{\rho}_{-j} \quad(j=0, \ldots, M) .
$$

The frequencies $\omega_{j}(j=-M, \ldots, M)$ can be extended with the period $2 \pi$ such that for example $\omega_{-M-1}:=-2 \pi+\omega_{M}$ and $\omega_{M+1}:=2 \pi-\omega_{M}$. We introduce the separation distance $q$ of the frequency set $\left\{\omega_{j}: j=0, \ldots, M\right\}$ by

$$
q:=\min \left\{\omega_{j+1}-\omega_{j} ; j=0, \ldots, M\right\} .
$$

Then we have $q M<\pi$. Let $N \in \mathbb{N}$ be an integer with $N \geq 2 M+$ 1. Assume that the sampled data $h_{k}:=f(k)(k=0, \ldots, 2 N)$ are given. Since $\omega_{M}<\pi$, we infer that the Nyquist condition is fulfilled (see [2, p. 183]). From the $2 N+1$ sampled data

Email addresses: potts@mathematik.tu-chemnitz.de (Daniel Potts), manfred.tasche@uni-rostock.de (Manfred Tasche) $h_{k}(k=0, \ldots, 2 N)$ we have to recover the positive integer $M$, the complex coefficients $\rho_{j}$, and the frequencies $\omega_{j} \in[0, \pi)$ $(j=0, \ldots, M)$ with (1.2) such that

$$
\sum_{j=-M}^{M} \rho_{j} \mathrm{e}^{\mathrm{i} \omega_{j} k}=h_{k} \quad(k=0, \ldots, 2 N) .
$$

By $N \geq 2 M+1$, the overmodeling factor $(2 N+1) /(2 M+1)$ is larger than 2 . Here $2 N+1$ is the number of sampled data and $2 M+1$ is the number of exponential terms. Overmodeling means that the corresponding overmodeling factor is larger than 1 . The above spectral analysis problem is a nonlinear inverse problem which can be simplified by original ideas of G.R. de Prony. But the classical Prony method is notorious for its sensitivity to noise such that numerous modifications were attempted to improve its numerical behavior. For the more general case with $\omega_{j} \in \mathbb{C}$ see e.g. [3] and the references therein. Our results are based on the paper [1] of G. Beylkin and L. Monzón. The nonlinear problem of finding the frequencies and coefficients can be split into two problems. To obtain the frequencies, we solve a singular value problem of the rectangular Hankel matrix $\mathbf{H}=(f(k+l))_{k, l=0}^{2 N-L, L}$ and find the frequencies via roots of a convenient polynomial of degree $L$, where $L$ denotes an a priori known upper bound of $2 M+1$. To obtain the coefficients, we use the frequencies to solve an overdetermined linear Vandermonde-type system. Note that the solution of the overdetermined linear Vandermonde-type system is closely related to the inverse nonequispaced fast Fourier transform studied recently by the authors [4]. In contrast to [1], we present an 
approximate Prony method (APM) by means of matrix perturbation theory such that we can describe the properties and the numerical behavior of the APM in detail. The first part of APM estimates the frequencies and the second part solves an overdetermined linear Vandermonde-type system in a stable way. We compare the first part of APM also with the known ESPRIT method.

In applications, perturbed values $\tilde{h}_{k} \in \mathbb{R}$ of the exact sampled data $h_{k}=f(k)$ are only known with the property

$$
\tilde{h}_{k}=h_{k}+e_{k}, \quad\left|e_{k}\right| \leq \varepsilon_{1} \quad(k=0, \ldots, 2 N),
$$

where the error terms $e_{k}$ are bounded by certain accuracy $\varepsilon_{1}>$ 0 . Also even if the sampled values $h_{k}$ are accurately determined, we still have a small roundoff error due to the use of floating point arithmetic. Furthermore we assume that $\left|\rho_{j}\right| \geq \varepsilon_{1}$ $(j=0, \ldots, M)$.

This paper is organized as follows. In Section 2, we sketch the classical Prony method. Then in Section 3, we generalize a first APM based on ideas of G. Beylkin and L. Monzón [1]. The Sections 4 and 5 form the core of this paper with new results on APM. Using matrix perturbation theory, we discuss the properties of small singular values and related right singular vectors of a real rectangular Hankel matrix formed by given noisy data. By means of the separation distance of the frequency set, we can describe the numerical behavior of the APM also for clustered frequencies, if we use overmodeling. Further we discuss the sensitivity of the APM to perturbation. Finally, various numerical examples are presented in Section 6.

\section{Classical Prony method}

The classical Prony method works with exact sampled data. Following an idea of G.R. de Prony from 1795 (see e.g. [5, pp. 303-310]), we regard the sampled data $h_{k}=f(k)(k=$ $0, \ldots, 2 N)$ as solution of a homogeneous linear difference equation with constant coefficients. If

$$
h_{k}=f(k)=\sum_{j=-M}^{M} \rho_{j}\left(\mathrm{e}^{\mathrm{i} \omega_{j}}\right)^{k}
$$

with (1.2) is a solution of certain homogeneous linear difference equation with constant coefficients, then $\mathrm{e}^{\mathrm{i} \omega_{j}}(j=-M, \ldots, M)$ must be zeros of the corresponding characteristic polynomial. Thus

$$
\begin{aligned}
P_{0}(z) & :=\prod_{j=-M}^{M}\left(z-\mathrm{e}^{\mathrm{i} \omega_{j}}\right)=(z-1) \prod_{j=1}^{M}\left(z^{2}-2 z \cos \omega_{j}+1\right) \\
& =\sum_{k=0}^{2 M+1} p_{k} z^{k} \quad(z \in \mathbb{C})
\end{aligned}
$$

with $p_{2 M+1}=-p_{0}=1$ is the monic characteristic polynomial of minimal degree. With the real coefficients $p_{k}(k=0, \ldots, 2 M+$ 1 ), we compose the homogeneous linear difference equation

$$
\sum_{l=0}^{2 M+1} x_{l+m} p_{l}=0 \quad(m=0,1, \ldots),
$$

which obviously has $P_{0}$ as characteristic polynomial. Consequently, (2.2) has the real general solution $x_{m}=$ $\sum_{j=-M}^{M} \rho_{j} \mathrm{e}^{\mathrm{i} \omega_{j} m} \quad(m=0,1, \ldots)$ with arbitrary coefficients $\rho_{0} \in$ $\mathbb{R}$ and $\rho_{j} \in \mathbb{C}(j=1, \ldots, M)$, where (1.2) is fulfilled. Then we determine $\rho_{j}(j=0, \ldots, M)$ in such a way that $x_{k} \approx h_{k}$ $(k=0, \ldots, 2 N)$. To this end, we compute the least squares solution of the overdetermined linear Vandermonde-type system

$$
\sum_{j=-M}^{M} \rho_{j} \mathrm{e}^{\mathrm{i} \omega_{j} k}=h_{k} \quad(k=0, \ldots, 2 N) .
$$

Let $L \in \mathbb{N}$ be a convenient upper bound of $2 M+1$, i.e., $2 M+1 \leq$ $L \leq N$. In applications, such an upper bound $L$ is mostly known a priori. If this is not the case, then one can choose $L=N$.

The idea of G.R. de Prony is based on the separation of the unknown frequencies $\omega_{j}$ from the unknown coefficients $\rho_{j}$ by means of a homogeneous linear difference equation (2.2). With the $2 N+1$ sampled data $h_{k} \in \mathbb{R}$ we form the rectangular Hankel matrix

$$
\mathbf{H}:=\left(h_{l+m}\right)_{l, m=0}^{2 N-L, L} \in \mathbb{R}^{(2 N-L+1) \times(L+1)} .
$$

Using the coefficients $p_{k}(k=0, \ldots, 2 M+1)$ of $(2.1)$, we construct the vector $\mathbf{p}:=\left(p_{k}\right)_{k=0}^{L}$, where $p_{2 M+2}=\ldots=p_{L}:=0$. By $\mathbf{S}:=\left(\delta_{k-l-1}\right)_{k, l=0}^{L}$ we denote the forward shift matrix, where $\delta_{k}$ is the Kronecker symbol.

Lemma 2.1 Let $L, M, N \in \mathbb{N}$ with $2 M+1 \leq L \leq N$ be given. Furthermore let $h_{k} \in \mathbb{R}$ be given by

$$
h_{k}=f(k)=\sum_{j=-M}^{M} \rho_{j} \mathrm{e}^{\mathrm{i} \omega_{j} k} \quad(k=0, \ldots, 2 N)
$$

with $\rho_{0} \in \mathbb{R} \backslash\{0\}, \rho_{j} \in \mathbb{C} \backslash\{0\}(j=1, \ldots, M), \omega_{0}=0<\omega_{1}<$ $\ldots<\omega_{M}<\pi$, where (1.2) is fulfilled.

Then the rectangular Hankel matrix (2.3) has the singular value 0 , where

$$
\operatorname{ker} \mathbf{H}=\operatorname{span}\left\{\mathbf{p}, \mathbf{S p}, \ldots, \mathbf{S}^{L-2 M-1} \mathbf{p}\right\}
$$

and

$$
\operatorname{dim}(\operatorname{ker} \mathbf{H})=L-2 M
$$

For shortness the proof is omitted. The Prony method is based on following

Lemma 2.2 Let $L, M, N \in \mathbb{N}$ with $2 M+1 \leq L \leq N$ be given. Let (2.4) be exact sampled data with $\rho_{0} \in \mathbb{R} \backslash\{0\}, \rho_{j} \in \mathbb{C} \backslash\{0\}$ $(j=1, \ldots, M)$ and $\omega_{0}=0<\omega_{1}<\ldots<\omega_{M}<\pi$, where (1.2) is fulfilled. Then the following assertions are equivalent:

(i) The polynomial

$$
P(z)=\sum_{k=0}^{L} u_{k} z^{k} \quad(z \in \mathbb{C})
$$

with real coefficients $u_{k}(k=0, \ldots, L)$ has $2 M+1$ different zeros $\mathrm{e}^{\mathrm{i} \omega_{j}}(j=-M, \ldots, M)$ on the unit circle.

(ii) 0 is a singular value of the real rectangular Hankel matrix (2.3) with a right singular vector $\mathbf{u}:=\left(u_{l}\right)_{l=0}^{L} \in \mathbb{R}^{L+1}$. 
The simple proof is omitted. Now we formulate Lemma 2.2 as algorithm:

\section{Algorithm 2.3 (Classical Prony Method)}

Input: $L, N \in \mathbb{N}(N \gg 1,3 \leq L \leq N, L$ is an upper bound of the number of exponentials), $h_{k}=f(k)(k=0, \ldots, 2 N), 0<\varepsilon \ll 1$.

1. Compute a right singular vector $\mathbf{u}=\left(u_{l}\right)_{l=0}^{L}$ corresponding to the singular value 0 of the exact rectangular Hankel matrix (2.3).

2. Form the corresponding polynomial (2.5) and evaluate all zeros $\mathrm{e}^{\mathrm{i} \omega_{j}}(j=0, \ldots, \tilde{M})$ with $\omega_{j} \in[0, \pi)$ and (1.2) lying on the unit circle. Note that $L \geq 2 \tilde{M}+1$.

3. Compute $\rho_{0} \in \mathbb{R}$ and $\rho_{j} \in \mathbb{C}(j=1, \ldots, \tilde{M})$ with (1.2) as least squares solution of the overdetermined linear Vandermonde-type system

$$
\sum_{j=-\tilde{M}}^{\tilde{M}} \rho_{j} \mathrm{e}^{\mathrm{i} \omega_{j} k}=h_{k} \quad(k=0, \ldots, 2 N) .
$$

4. Delete all the pairs $\left(\omega_{l}, \rho_{l}\right)(l \in\{1, \ldots, \tilde{M}\})$ with $\left|\rho_{l}\right| \leq \varepsilon$ and denote the remaining set by $\left\{\left(\omega_{j}, \rho_{j}\right): j=1, \ldots, M\right\}$ with $M \leq \tilde{M}$.

Output: $M \in \mathbb{N}, \rho_{0} \in \mathbb{R}, \rho_{j} \in \mathbb{C}, \omega_{j} \in(0, \pi)(j=1, \ldots, M)$.

Remark 2.4 We can determine all roots of the polynomial (2.5) with $u_{L}=1$ by computing the eigenvalues of the companion matrix

$$
\mathbf{U}:=\left(\begin{array}{ccccc}
0 & 0 & \ldots & 0 & -u_{0} \\
1 & 0 & \ldots & 0 & -u_{1} \\
0 & 1 & \ldots & 0 & -u_{2} \\
\vdots & \vdots & & \vdots & \vdots \\
0 & 0 & \ldots & 1 & -u_{L-1}
\end{array}\right) \in \mathbb{R}^{L \times L}
$$

This follows immediately from the fact $P(z)=\operatorname{det}(z \mathbf{I}-\mathbf{U})$. Note that we consider a rectangular Hankel matrix (2.3) with only $L$ columns in order to determine the zeros of a polynomial (2.5) with relatively low degree L (see step 2 of Algorithm 2.3).

Remark 2.5 Let $N>2 M+1$. If one knows $M$ or a good approximation of $M$, then one can use the following least squares Prony method, see e.g. [6]. Since the leading coefficient $p_{2 M+1}$ of the characteristic polynomial $P_{0}$ is equal to $1,(2.2)$ gives rise to the overdetermined linear system

$$
\begin{aligned}
\sum_{l=0}^{2 M} h_{l+m} p_{l} & =-h_{2 M+1+m} p_{2 M+1} \\
& =-h_{2 M+1+m} \quad(m=0, \ldots, 2 N-2 M-1),
\end{aligned}
$$

which can be solved by a least squares method. See also the relation to the classic Yule-Walker system [7].

\section{Approximate Prony method}

The classical Prony method is known to perform poorly when noisy data are given. Therefore numerous modifications were attempted to improve the numerical behavior of the classical Prony method. In practice, only perturbed values $\tilde{h}_{k}:=h_{k}+e_{k}$ $(k=0, \ldots, 2 N)$ of the exact sampled data $h_{k}=f(k)$ are known. Here we assume that $\left|e_{k}\right| \leq \varepsilon_{1}$ with certain accuracy $\varepsilon_{1}>0$. Then the rectangular error Hankel matrix

$$
\mathbf{E}:=\left(e_{k+l}\right)_{k, l=0}^{2 N-L, L}
$$

has a small spectral norm satisfying

$$
\begin{aligned}
\|\mathbf{E}\|_{2} & \leq \sqrt{\|\mathbf{E}\|_{1}\|\mathbf{E}\|_{\infty}} \leq \sqrt{(L+1)(2 N-L+1)} \varepsilon_{1} \\
& \leq(N+1) \varepsilon_{1} .
\end{aligned}
$$

Thus the perturbed Hankel matrix can be represented by

$$
\tilde{\mathbf{H}}:=\left(\tilde{h}_{k+l}\right)_{k, l=0}^{2 N-L, L}=\mathbf{H}+\mathbf{E} \in \mathbb{R}^{(2 N-L+1) \times(L+1)} .
$$

Using an analog of the Theorem of H. Weyl (see [8, p. 419]) and Lemma 2.1, we receive bounds for small singular values of $\tilde{\mathbf{H}}$. More precisely, $L-2 M$ singular values of $\tilde{\mathbf{H}}$ are contained in $\left[0,\|\mathbf{E}\|_{2}\right]$, if each nonzero singular value of $\mathbf{H}$ is larger than $2\|\mathbf{E}\|_{2}$. In the following we use this property and evaluate a small singular value $\tilde{\sigma}\left(0<\tilde{\sigma} \leq \varepsilon_{2} \ll 1\right)$ and corresponding right and left singular vectors of the perturbed rectangular Hankel matrix $\tilde{\mathbf{H}}$.

Recently, a very interesting approach is described by G. Beylkin and L. Monzón [1], where the more general problem of approximation by exponential sums is considered. In contrast to [1], we consider a perturbed rectangular Hankel matrix (3.2).

Theorem 3.1 (cf. [1]) Let $\tilde{\sigma} \in\left(0, \varepsilon_{2}\right]\left(0<\varepsilon_{2} \ll 1\right)$ be a small singular value of the perturbed rectangular Hankel matrix (3.2) with a right singular vector $\tilde{\mathbf{u}}=\left(\tilde{u}_{k}\right)_{k=0}^{L} \in \mathbb{R}^{L+1}$ and a left singular vector $\tilde{\mathbf{v}}=\left(\tilde{v}_{k}\right)_{k=0}^{2 N-L} \in \mathbb{R}^{2 N-L+1}$. Assume that the polynomial

$$
\tilde{P}(z)=\sum_{k=0}^{L} \tilde{u}_{k} z^{k} \quad(z \in \mathbb{C}),
$$

has L pairwise distinct zeros $\tilde{w}_{n} \in \mathbb{C}(n=1, \ldots, L)$. Further let $K>2 N$.

Then there exists a unique vector $\left(a_{n}\right)_{n=1}^{L} \in \mathbb{C}^{L}$ such that

$$
\tilde{h}_{k}=\sum_{n=1}^{L} a_{n} \tilde{w}_{n}^{k}+\tilde{\sigma} d_{k} \quad(k=0, \ldots, 2 N),
$$

where the vector $\left(d_{k}\right)_{k=0}^{K-1} \in \mathbb{C}^{K}$ is defined as follows. Let

$$
\begin{aligned}
\hat{u}_{l} & :=\sum_{k=0}^{L-1} \tilde{u}_{k} \mathrm{e}^{-2 \pi \mathrm{i} k l / K} \quad(l=0, \ldots, K-1), \\
\hat{v}_{l} & :=\sum_{k=0}^{2 N-L} \tilde{v}_{k} \mathrm{e}^{-2 \pi \mathrm{i} k l / K} \quad(l=0, \ldots, K-1), \\
\hat{d}_{l} & := \begin{cases}\hat{v}_{l} / \overline{\hat{u}}_{l} & \text { if } \hat{u}_{l} \neq 0, \\
1 & \text { if } \hat{u}_{l}=0 .\end{cases}
\end{aligned}
$$


Then

$$
d_{k}:=\frac{1}{K} \sum_{l=0}^{K-1} \hat{d}_{l} \mathrm{e}^{2 \pi \mathrm{i} k l / K} \quad(k=0, \ldots, K-1) .
$$

The vector $\left(a_{n}\right)_{n=1}^{L}$ can be computed as solution of the linear Vandermonde system

$$
\sum_{n=1}^{L} a_{n} \tilde{w}_{n}^{k}=\tilde{h}_{k}-\tilde{\sigma} d_{k} \quad(k=0, \ldots, L-1)
$$

Furthermore, if

$$
\left|\hat{v}_{l}\right| \leq \gamma\left|\hat{u}_{l}\right| \quad(l=0, \ldots, K-1)
$$

for certain constant $\gamma>0$, then

$$
\begin{gathered}
\sum_{k=0}^{K-1}\left|d_{k}\right|^{2} \leq \gamma^{2}, \\
\left|h_{k}-\sum_{n=1}^{L} a_{n} \tilde{w}_{n}^{k}\right| \leq \varepsilon_{1}+\gamma \varepsilon_{2} \quad(k=0, \ldots, 2 N) .
\end{gathered}
$$

Proof. 1. From $\tilde{\mathbf{H}} \tilde{\mathbf{u}}=\tilde{\sigma} \tilde{\mathbf{v}}\left(\right.$ and $\left.\tilde{\mathbf{H}}^{\mathrm{T}} \tilde{\mathbf{v}}=\tilde{\sigma} \tilde{\mathbf{u}}\right)$ it follows that

$$
\sum_{l=0}^{L} \tilde{h}_{l+m} \tilde{u}_{l}=\tilde{\sigma} \tilde{v}_{m} \quad(m=0, \ldots, 2 N-L) .
$$

We set $\tilde{u}_{n}:=0(n=L+1, \ldots, K-1)$ and extend the vector $\left(\tilde{u}_{n}\right)_{n=0}^{K-1}$ to the $K$-periodic sequence $\left(\tilde{u}_{n}\right)_{n=0}^{\infty}$ by $\tilde{u}_{n+j K}:=\tilde{u}_{n}$ $(j \in \mathbb{N} ; n=0, \ldots, K-1)$. Analogously, we set $\tilde{v}_{n}:=0$ $(n=2 N-L+1, \ldots, K-1)$ and form the $K$-periodic sequence $\left(\tilde{v}_{n}\right)_{n=0}^{\infty}$ by $\tilde{v}_{n+j K}:=\tilde{v}_{n}(j \in \mathbb{N} ; n=0, \ldots, K-1)$. Then we consider the inhomogeneous linear difference equation with constant coefficients

$$
\sum_{n=0}^{L} x_{k+n} \tilde{u}_{n}=\tilde{\sigma} \tilde{v}_{k} \quad(k=0,1, \ldots)
$$

under the initial conditions $x_{k}=\tilde{h}_{k}(k=0, \ldots, L-1)$. By assumption, the polynomial $\tilde{P}$ possesses $L$ pairwise different zeros such that $\tilde{u}_{L} \neq 0$. Hence the linear difference equation (3.10) has the order $L$. Thus the above initial value problem of (3.10) is uniquely solvable.

2. As known, the general solution of (3.10) is the sum of the general solution of the corresponding homogeneous linear difference equation

$$
\sum_{n=0}^{L} x_{k+n} \tilde{u}_{n}=0 \quad(k=0,1, \ldots)
$$

and a special solution $\tilde{\sigma}\left(d_{k}\right)_{k=0}^{\infty}$ of (3.10). The characteristic equation of (3.10) is $\tilde{P}(z)=0$ and has the pairwise different solutions $\tilde{w}_{n} \in \mathbb{C}(n=1, \ldots, L)$ by assumption. Thus the general solution of (3.10) reads as follows

$$
x_{k}=\sum_{n=1}^{L} a_{n} \tilde{w}_{n}^{k}+\tilde{\sigma} d_{k} \quad(k=0,1, \ldots)
$$

with arbitrary constants $a_{n} \in \mathbb{C}$. By the initial conditions $x_{k}=$ $\tilde{h}_{k}(k=0, \ldots, L-1)$, the constants $a_{n}$ are the unique solutions of the linear Vandermonde system (3.8). Note that $\left(x_{k}\right)_{k=0}^{\infty}$ is not an $K$-periodic sequence in general.

3. By $\tilde{u}_{L} \neq 0$, we can extend the given data vector $\left(\tilde{h}_{l}\right)_{l=0}^{2 N}$ to a sequence $\left(\tilde{h}_{l}\right)_{l=0}^{\infty}$ in the following way

$$
\tilde{h}_{2 N+k}:=-\frac{1}{\tilde{u}_{L}} \sum_{n=0}^{L-1} \tilde{h}_{2 N-L+k+n} \tilde{u}_{n}+\frac{\tilde{\sigma}}{\tilde{u}_{L}} \tilde{v}_{2 N-L+k} \quad(k=1,2, \ldots) .
$$

Thus we receive

$$
\sum_{n=0}^{L} \tilde{h}_{k+n} \tilde{u}_{n}=\tilde{\sigma} \tilde{v}_{k} \quad(k=0,1, \ldots),
$$

i.e., the sequence $\left(\tilde{h}_{l}\right)_{l=0}^{\infty}$ is also a solution of (3.10) with the initial conditions $x_{k}=\tilde{h}_{k}(k=0, \ldots, L-1)$. Since this initial value problem is uniquely solvable, it follows for all $k=0,1 \ldots$ that

$$
\tilde{h}_{k}=x_{k}=\sum_{n=1}^{L} a_{n} \tilde{w}_{n}^{k}+\tilde{\sigma} d_{k} .
$$

4. By means of discrete Fourier transform of length $K$ $(\mathrm{DFT}(K))$, we can construct a special $K$-periodic solution $\tilde{\sigma}\left(d_{k}\right)_{k=0}^{\infty}$ of (3.10). Then for the $K$-periodic sequences $\left(d_{k}\right)_{k=0}^{\infty}$, $\left(\tilde{u}_{k}\right)_{k=0}^{\infty}$, and $\left(\tilde{v}_{k}\right)_{k=0}^{\infty}$ we obtain

$$
\sum_{n=0}^{L} d_{k+n} \tilde{u}_{n}=\sum_{n=0}^{K-1} d_{k+n} \tilde{u}_{n}=\tilde{v}_{k} \quad(k=0, \ldots, K-1),
$$

where

$$
\left(\sum_{n=0}^{K-1} d_{k+n} \tilde{u}_{n}\right)_{k=0}^{\infty}
$$

is the $K$-periodic correlation of $\left(\tilde{u}_{k}\right)_{k=0}^{\infty}$ with respect to $\left(d_{k}\right)_{k=0}^{\infty}$. Introducing the following entries (3.4), (3.5), and

$$
\hat{d}_{l}:=\sum_{k=0}^{K-1} d_{k} \mathrm{e}^{-2 \pi \mathrm{i} k l / K} \quad(l=0, \ldots, K-1),
$$

from (3.12) it follows by $\operatorname{DFT}(K)$ that

$$
\hat{d}_{l} \overline{\hat{u}}_{l}=\hat{v}_{l} \quad(l=0, \ldots, K-1) .
$$

Note that $\hat{u}_{l}=0$ implies $\hat{v}_{l}=0$. Hence we obtain (3.6) with $\left|\hat{d}_{l}\right| \leq \gamma(l=0, \ldots, K-1)$. In the case $L=N$, one can show that $\gamma=1$. Using inverse $\operatorname{DFT}(K)$, we receive (3.7). By Parseval equation we see that

$$
\sum_{k=0}^{K-1}\left|d_{k}\right|^{2}=\frac{1}{K} \sum_{l=0}^{K-1}\left|\hat{d}_{l}\right|^{2} \leq \gamma^{2}
$$

and hence $\left|d_{k}\right| \leq \gamma(k=0, \ldots, K-1)$. Then for $k=0, \ldots, K-1$ we can estimate

$$
\begin{aligned}
\left|h_{k}-\sum_{n=1}^{L} a_{n} \tilde{w}_{n}^{k}\right| & \leq\left|h_{k}-\tilde{h}_{k}\right|+\left|\tilde{h}_{k}-\sum_{n=1}^{L} a_{n} \tilde{w}_{n}^{k}\right| \\
& \leq \varepsilon_{1}+\gamma \tilde{\sigma} \leq \varepsilon_{1}+\gamma \varepsilon_{2} .
\end{aligned}
$$

This completes the proof. 
Remark 3.2 This Theorem 3.1 yields a different representation for each $K>2 N$ even though $\tilde{w}_{n}$ and $\tilde{\sigma}$ remain the same. If $K$ is chosen as power of 2 , then the entries $\hat{u}_{l}, \hat{v}_{l}$ and $d_{k}$ $(l, k=0, \ldots, K-1)$ can be computed by fast Fourier transforms. Note that the least squares solution $\left(\tilde{b}_{n}\right)_{n=1}^{L}$ of the overdetermined linear Vandermonde-type system

$$
\sum_{n=1}^{L} \tilde{b}_{n} \tilde{w}_{n}^{k}=\tilde{h}_{k} \quad(k=0, \ldots, 2 N)
$$

has an error with Euclidean norm less than $\gamma \varepsilon_{2}$, since

$$
\begin{aligned}
& \sum_{k=0}^{2 N}\left|\tilde{h}_{k}-\sum_{n=1}^{L} \tilde{b}_{n} \tilde{w}_{n}^{k}\right|^{2} \leq \sum_{k=0}^{2 N}\left|\tilde{h}_{k}-\sum_{n=1}^{L} a_{n} \tilde{w}_{n}^{k}\right|^{2} \\
& =\sum_{k=0}^{2 N}\left|\tilde{\sigma} d_{k}\right|^{2} \leq \tilde{\sigma}^{2} \sum_{k=0}^{K-1}\left|d_{k}\right|^{2} \leq\left(\gamma \varepsilon_{2}\right)^{2}
\end{aligned}
$$

By Remark 3.2 an algorithm of the first APM (cf. [1]) reads as follows:

\section{Algorithm 3.3 (APM 1)}

Input: $L, N \in \mathbb{N}(3 \leq L \leq N, L$ is an upper bound of the number of exponentials), $\tilde{h}_{k} \in \mathbb{R}(k=0, \ldots, 2 N)$, accuracies $\varepsilon_{1}, \varepsilon_{2}>0$.

1. Compute a small singular value $\tilde{\sigma} \in\left(0, \varepsilon_{2}\right]$ and corresponding right and left singular vectors $\tilde{\mathbf{u}}=\left(\tilde{u}_{l}\right)_{l=0}^{L} \in \mathbb{R}^{L+1}$, $\tilde{\mathbf{v}}=\left(\tilde{v}_{l}\right)_{l=0}^{2 N-L} \in \mathbb{R}^{2 N-L+1}$ of the perturbed rectangular Hankel matrix (3.2).

2. Determine all zeros $\tilde{w}_{n} \in \mathbb{C}(n=1, \ldots, L)$ of the corresponding polynomial (3.3). Assume that all the zeros of $\tilde{P}$ are simple.

3. Determine the least squares solution $\left(\tilde{b}_{n}\right)_{n=1}^{L} \in \mathbb{C}^{L}$ of the overdetermined linear Vandermonde-type system (3.13).

4. Denote by $\left(w_{j}, \rho_{j}\right)(j=-M, \ldots, M)$ all that pairs $\left(\tilde{w}_{k}, \tilde{b}_{k}\right)$ $(k=1, \ldots, L)$ with the properties $\left|\tilde{w}_{k}\right| \approx 1$ and $\left|\tilde{b}_{k}\right| \geq \varepsilon_{1}$. Assume that $\arg w_{j} \in[0, \pi)$ and $w_{j}=\bar{w}_{-j}(j=0, \ldots, M)$. Set $\omega_{j}:=\arg w_{j}>0(j=1, \ldots, M)$ and $\omega_{0}:=0$.

Output: $M \in \mathbb{N}, \rho_{j} \in \mathbb{C}, \omega_{j} \in[0, \pi)(j=0, \ldots, M)$.

Similarly as in [1], we are not interested in exact representations of the sampled values

$$
\tilde{h}_{k}=\sum_{n=1}^{L} \tilde{b}_{n} \tilde{w}_{n}^{k} \quad(k=0, \ldots, 2 N)
$$

but rather in approximate representations

$$
\left|\tilde{h}_{k}-\sum_{j=-M}^{M} \rho_{j} \mathrm{e}^{\mathrm{i} \omega_{j} k}\right| \leq \tilde{\varepsilon} \quad(k=0, \ldots, 2 N)
$$

for very small accuracy $\tilde{\varepsilon}>0$ and minimal number $M$ of nontrivial summands. This fact explains the name of our method too.

\section{Approximate Prony method based on NFFT}

Now we present a second new approximate Prony method by means of matrix perturbation theory. First we introduce the rectangular Vandermonde-type matrix

$$
\mathbf{V}:=\left(\mathrm{e}^{\mathrm{i} k \omega_{j}}\right)_{k=0, j=-M}^{2 N, M} \in \mathbb{C}^{(2 N+1) \times(2 M+1)} .
$$

Note that $\mathbf{V}$ is also a nonequispaced Fourier matrix (see [9, 10, 4]). We discuss the properties of $\mathbf{V}$. Especially, we show that $\mathbf{V}$ is left-invertible and estimate the spectral norm of its left inverse. For these results, the separation distance (1.3) plays a crucial role.

Lemma 4.1 Let $M, N \in \mathbb{N}$ with $N \geq 2 M+1$ given. Furthermore let $\omega_{0}=0<\omega_{1}<\ldots<\omega_{M}<\pi$ with a separation distance

$$
q>\frac{1}{N+1} \frac{\pi^{2}}{\sqrt{3}}
$$

be given. Let (1.2) be fulfilled. Let $\mathbf{D}:=\operatorname{diag}(1-|k| /(N+1))_{k=-N}^{N}$ be a diagonal matrix.

Then for arbitrary $\mathbf{r} \in \mathbb{C}^{2 M+1}$, the following inequality

$\left(N+1-\frac{\pi^{4}}{3 q^{2}(N+1)}\right)\|\mathbf{r}\|^{2} \leq\left\|\mathbf{D}^{1 / 2} \mathbf{V r}\right\|_{2}^{2} \leq\left(N+1+\frac{\pi^{4}}{3 q^{2}(N+1)}\right)\|\mathbf{r}\|^{2}$

is fulfilled. Further

$$
\mathbf{L}:=\left(\mathbf{V}^{\mathrm{H}} \mathbf{D V}\right)^{-1} \mathbf{V}^{\mathrm{H}} \mathbf{D}
$$

is a left inverse of (4.1) and the squared spectral norm of $\mathbf{L}$ can be estimated by

$$
\|\mathbf{L}\|_{2}^{2} \leq \frac{3 q^{2}(N+1)}{3 q^{2}(N+1)^{2}-\pi^{4}} .
$$

Proof. 1. The rectangular Vandermonde-type matrix $\mathbf{V} \in$ $\mathbb{C}^{(2 N+1) \times(2 M+1)}$ has full rank $2 M+1$, since its submatrix

$$
\left(\mathrm{e}^{\mathrm{i} k \omega_{j}}\right)_{k=0, j=-M}^{2 M, M}
$$

is a regular Vandermonde matrix. Hence we infer that $\mathbf{V}^{\mathrm{H}} \mathbf{D V}$ is Hermitian and positive definite such that all eigenvalues of $\mathbf{V}^{\mathrm{H}} \mathbf{D V}$ are positive.

2. We introduce the $2 \pi$-periodic Fejér kernel $F_{N}$ by

$$
F_{N}(x):=\sum_{k=-N}^{N}\left(1-\frac{|k|}{N+1}\right) \mathrm{e}^{\mathrm{i} k x} .
$$

Then we obtain

$$
\left(\mathbf{V}^{\mathrm{H}} \mathbf{D V}\right)_{j, l}=\mathrm{e}^{-\mathrm{i} N \omega_{j}} F_{N}\left(\omega_{j}-\omega_{l}\right) \mathrm{e}^{\mathrm{i} N \omega_{l}} \quad(j, l=-M, \ldots, M)
$$

for the $(j, l)$-th entry of the matrix $\mathbf{V}^{\mathrm{H}} \mathbf{D V}$. We use Gershgorin's Disk Theorem (see [8, p. 344]) such that for an arbitrary eigenvalue $\lambda$ of the matrix $\mathbf{V}^{\mathrm{H}} \mathbf{D V}$ we preserve the estimate (see also [11, Theorem 4.1])

$$
\begin{aligned}
& \left|\lambda-F_{N}(0)\right|=|\lambda-N-1| \\
& \leq \max \left\{\sum_{\substack{j=-M \\
j \neq l}}^{M}\left|F_{N}\left(\omega_{j}-\omega_{l}\right)\right| ; l=-M, \ldots, M\right\} .
\end{aligned}
$$


3. Now we estimate the right-hand side of (4.5). As known, the Fejér kernel $F_{N}$ can be written in the form

$$
0 \leq F_{N}(x)=\frac{1}{N+1}\left(\frac{\sin ((N+1) x / 2)}{\sin (x / 2)}\right)^{2} .
$$

Thus we obtain the estimate

$$
\sum_{\substack{j=-M \\ j \neq l}}^{M}\left|F_{N}\left(\omega_{j}-\omega_{l}\right)\right| \leq \frac{1}{N+1} \sum_{\substack{j=-M \\ j \neq l}}^{M}\left|\sin \left(\left(\omega_{j}-\omega_{l}\right) / 2\right)\right|^{-2}
$$

for $l \in\{-M, \ldots, M\}$. In the case $l=0$, we use (1.2), $q M<\pi$, $\omega_{j} \geq j q(j=1, \ldots, M)$ and

$$
\sin x \geq \frac{2}{\pi} x \quad(x \in[0, \pi / 2])
$$

and then we estimate the above sum by

$$
\begin{aligned}
\sum_{\substack{j=-M \\
j \neq 0}}^{M}\left|\sin \left(\omega_{j} / 2\right)\right|^{-2} & =2 \sum_{j=1}^{M}\left(\sin \left(\omega_{j} / 2\right)\right)^{-2} \leq 2 \pi^{2} \sum_{j=1}^{M} \omega_{j}^{-2} \\
& \leq \frac{2 \pi^{2}}{q^{2}} \sum_{j=1}^{M} j^{-2}<\frac{\pi^{4}}{3 q^{2}} .
\end{aligned}
$$

By similar arguments, we obtain

$$
\sum_{\substack{j=-M \\ j \neq l}}^{M}\left|\sin \left(\left(\omega_{j}-\omega_{l}\right) / 2\right)\right|^{-2}<\frac{\pi^{4}}{3 q^{2}}
$$

in case $l \in\{ \pm 1, \ldots, \pm M\}$. Note that we use the $2 \pi$-periodization of the frequencies $\omega_{j}(j=-M, \ldots, M)$ such that

$$
\left|\sin \left(\left(\omega_{j}-\omega_{l}\right) / 2\right)\right|=\left|\sin \left( \pm \pi+\left(\omega_{j}-\omega_{l}\right) / 2\right)\right|
$$

Hence it follows in each case that

$$
|\lambda-N-1|<\frac{\pi^{4}}{3 q^{2}(N+1)} .
$$

4. Let $\lambda_{\min }$ and $\lambda_{\max }$ be the smallest and largest eigenvalue of $\mathbf{V}^{\mathrm{H}} \mathbf{D V}$, respectively. Using (4.6), we receive

$N+1-\frac{\pi^{4}}{3 q^{2}(N+1)} \leq \lambda_{\min } \leq N+1 \leq \lambda_{\max } \leq N+1+\frac{\pi^{4}}{3 q^{2}(N+1)}$,

where by assumption

$$
N+1-\frac{\pi^{4}}{3 q^{2}(N+1)}>0
$$

Using the variational characterization of the Rayleigh-Ritz ratio for the Hermitian matrix $\mathbf{V}^{\mathrm{H}} \mathbf{D V}$ (see [8, p. 176]), we obtain for arbitrary $\mathbf{r} \in \mathbb{C}^{2 M+1}$ that

$$
\lambda_{\min }\|\mathbf{r}\|^{2} \leq\left\|\mathbf{D}^{1 / 2} \mathbf{V} \mathbf{r}\right\|^{2} \leq \lambda_{\max }\|\mathbf{r}\|^{2} .
$$

From

$$
\mathbf{L} \mathbf{V}=\left(\mathbf{V}^{\mathrm{H}} \mathbf{D V}\right)^{-1} \mathbf{V}^{\mathrm{H}} \mathbf{D}^{1 / 2} \mathbf{D}^{1 / 2} \mathbf{V}=\mathbf{I}
$$

it follows that (4.3) is a left inverse of $\mathbf{V}$ and that the singular values of $\mathbf{D}^{1 / 2} \mathbf{V}$ lie in $\left[\sqrt{\lambda_{\min }}, \sqrt{\lambda_{\max }}\right]$. Hence we obtain for the squared spectral norm of the left inverse $\mathbf{L}$ (see also [4, Theorem 4.2])

$$
\begin{aligned}
\|\mathbf{L}\|_{2}^{2} & \leq\left\|\left(\mathbf{V}^{\mathrm{H}} \mathbf{D V}\right)^{-1} \mathbf{V}^{\mathrm{H}} \mathbf{D}^{1 / 2}\right\|_{2}^{2}\left\|\mathbf{D}^{1 / 2}\right\|_{2}^{2} \leq \lambda_{\text {min }}^{-1} \\
& \leq \frac{3 q^{2}(N+1)}{3 q^{2}(N+1)^{2}-\pi^{4}} .
\end{aligned}
$$

This completes the proof.

Corollary 4.2 Let $M \in \mathbb{N}$ be given. Furthermore let $\omega_{0}=0<$ $\omega_{1}<\ldots<\omega_{M}<\pi$ with separation distance $q$. Let (1.2) be fulfilled.

Then for each $N \in \mathbb{N}$ with

$$
N>\frac{\pi^{2}}{q}
$$

the squared spectral norm of (4.3) satisfies

$$
\|\mathbf{L}\|_{2}^{2} \leq \frac{3}{2 N+2} .
$$

Proof. By (4.7) and $q M<\pi$, we see immediately that

$$
2 M+1<\frac{2 \pi}{q}+1<\frac{\pi^{2}}{q}<N
$$

Further from (4.7) it follows that

$$
q>\frac{\pi^{2}}{N}>\frac{\pi^{2}}{\sqrt{3}(N+1)} .
$$

Thus the assumptions of Lemma 4.1 are fulfilled. Using the upper estimate (4.4) of $\|\mathbf{L}\|_{2}^{2}$ and (4.7), we obtain the result.

Corollary 4.3 Let $M \in \mathbb{N}$ be given. Furthermore let $\omega_{0}=0<$ $\omega_{1}<\ldots<\omega_{M}<\pi$ with separation distance $q$. Let (1.2) be fulfilled.

Then the squared spectral norm of the Vandermonde-type matrix $\mathbf{V}$ is bounded, i.e.,

$$
\|\mathbf{V}\|_{2}^{2} \leq 2 N+1+\frac{2 \pi}{q}\left(1+\ln \frac{\pi}{q}\right) .
$$

Proof. We follow the lines of the proof of Lemma 4.1 with the trivial diagonal matrix $\mathbf{D}:=\operatorname{diag}(1)_{k=-N}^{N}$. Instead of the Fejér kernel $F_{N}$ we use the modified Dirichlet kernel

$$
D_{N}(x):=\sum_{k=0}^{2 N} \mathrm{e}^{\mathrm{i} k x}=\mathrm{e}^{\mathrm{i} N x} \frac{\sin ((2 N+1) x / 2)}{\sin (x / 2)}
$$

and we obtain for the $(j, l)$-th entry of $\mathbf{V}^{\mathrm{H}} \mathbf{V}$

$$
\left(\mathbf{V}^{\mathrm{H}} \mathbf{V}\right)_{j, l}=D_{N}\left(\omega_{j}-\omega_{l}\right) \quad(j, l=-M, \ldots, M) .
$$

We proceed with

$$
\begin{aligned}
& \left|\lambda-D_{N}(0)\right|=|\lambda-(2 N+1)| \\
& \leq \max \left\{\sum_{\substack{j=-M \\
j \neq l}}^{M}\left|D_{N}\left(\omega_{j}-\omega_{l}\right)\right| ; l=-M, \ldots, M\right\},
\end{aligned}
$$


use the estimate

$$
\sum_{\substack{j=-M \\ j \neq l}}^{M}\left|D_{N}\left(\omega_{j}-\omega_{l}\right)\right| \leq \sum_{\substack{j=-M \\ j \neq l}}^{M}\left|\sin \left(\left(\omega_{j}-\omega_{l}\right) / 2\right)\right|^{-1}
$$

and infer

$$
\begin{aligned}
& \sum_{\substack{j=-M \\
j \neq 0}}^{M}\left|\sin \left(\omega_{j} / 2\right)\right|^{-1}=2 \sum_{j=1}^{M}\left(\sin \left(\omega_{j} / 2\right)\right)^{-1} \leq 2 \pi \sum_{j=1}^{M} \omega_{j}^{-1} \\
& \leq \frac{2 \pi}{q} \sum_{j=1}^{M} j^{-1}<\frac{2 \pi}{q}(1+\ln M)<\frac{2 \pi}{q}\left(1+\ln \frac{\pi}{q}\right) .
\end{aligned}
$$

Finally we obtain for the largest eigenvalue of $\mathbf{V}^{\mathrm{H}} \mathbf{V}$

$$
\lambda_{\max }\left(\mathbf{V}^{\mathrm{H}} \mathbf{V}\right) \leq 2 N+1+\frac{2 \pi}{q}\left(1+\ln \frac{\pi}{q}\right)
$$

and hence the assertion.

Let $L, M, N \in \mathbb{N}$ with $2 M+1 \leq L \leq N$ be given, where $L$ is even. Now we consider a special submatrix $\mathbf{V}_{1}$ of $\mathbf{V}$ defined by

$$
\mathbf{V}_{1}:=\left(\mathrm{e}^{\mathrm{i} k \omega_{j}}\right)_{k=0, j=-M}^{2 N-L, M} \in \mathbb{C}^{(2 N-L+1) \times(2 M+1)} .
$$

The matrix $\mathbf{V}_{1}$ has full rank $2 M+1$ (see step 1 of Lemma 4.1). Hence $\mathbf{V}_{1}^{\mathrm{H}} \mathbf{D}_{1} \mathbf{V}_{1}$ is Hermitian and positive definite, where

$$
\mathbf{D}_{1}:=\operatorname{diag}\left(1-\frac{|k|}{N+1-L / 2}\right)_{k=-N+L / 2}^{N-L / 2} .
$$

Using Lemma 4.1, the matrix $\mathbf{V}_{1}$ has a left inverse

$$
\mathbf{L}_{1}:=\left(\mathbf{V}_{1}^{\mathrm{H}} \mathbf{D}_{1} \mathbf{V}_{1}\right)^{-1} \mathbf{V}_{1}^{\mathrm{H}} \mathbf{D}_{1}
$$

Theorem 4.4 Let $L, M, N \in \mathbb{N}$ with $2 M+1 \leq L \leq N$ be given, where $L$ is even. Furthermore let $h_{k} \in \mathbb{R}$ be given as in (2.4) with $\rho_{0} \in \mathbb{R} \backslash\{0\}, \rho_{j} \in \mathbb{C} \backslash\{0\}(j=1, \ldots, M)$, and $\omega_{0}=$ $0<\omega_{1}<\ldots<\omega_{M}<\pi$. Let (1.2) be fulfilled. Assume that the perturbed Hankel matrix (3.2) has $\tilde{\sigma} \in\left(0, \varepsilon_{2}\right]$ as singular value with the corresponding normed right and left singular vectors $\tilde{\mathbf{u}}=\left(\tilde{u}_{n}\right)_{n=0}^{L} \in \mathbb{R}^{L+1}$ and $\tilde{\mathbf{v}}=\left(\tilde{v}_{m}\right)_{m=0}^{2 N-L} \in \mathbb{R}^{2 N-L+1}$. Let $\tilde{P}$ be the polynomial (3.3) related to $\tilde{\mathbf{u}}$.

Then the values $\tilde{P}\left(\mathrm{e}^{\mathrm{i} \omega_{j}}\right)(j=-M, \ldots, M)$ fulfill the estimate

$$
\rho_{0}^{2} \tilde{P}(1)^{2}+2 \sum_{j=1}^{M}\left|\rho_{j}\right|^{2}\left|\tilde{P}\left(\mathrm{e}^{\mathrm{i} \omega_{j}}\right)\right|^{2} \leq\left(\varepsilon_{2}+\|\mathbf{E}\|_{2}\right)^{2}\left\|\mathbf{L}_{1}\right\|_{2}^{2} .
$$

Proof. 1. By assumption we have $\tilde{\mathbf{H}} \tilde{\mathbf{u}}=\tilde{\sigma} \tilde{\mathbf{v}}$, i.e.,

$$
\sum_{l=0}^{L} \tilde{h}_{l+m} \tilde{u}_{l}=\tilde{\sigma} \tilde{v}_{m} \quad(m=0, \ldots, 2 N-L) .
$$

Applying (2.4) and $\tilde{h}_{k}=h_{k}+e_{k}$, we obtain for $m=0, \ldots, 2 N-L$

$$
\sum_{j=-M}^{M} \rho_{j} \tilde{P}\left(\mathrm{e}^{\mathrm{i} \omega_{j}}\right) \mathrm{e}^{\mathrm{i} m \omega_{j}}=\tilde{\sigma} \tilde{v}_{m}-\sum_{l=0}^{L} e_{l+m} \tilde{u}_{l} .
$$

2. Using the matrix-vector notation of (4.8) with the rectangular Vandermonde-type matrix $\mathbf{V}_{1}$, we receive

$$
\mathbf{V}_{1}\left(\rho_{j} \tilde{P}\left(\mathrm{e}^{\mathrm{i} \omega_{j}}\right)\right)_{j=-M}^{M}=\tilde{\sigma} \tilde{\mathbf{v}}-\mathbf{E} \tilde{\mathbf{u}}
$$

The matrix $\mathbf{V}_{1}$ has a left inverse $\mathbf{L}_{1}=\left(\mathbf{V}_{1}^{\mathrm{H}} \mathbf{D}_{1} \mathbf{V}_{1}\right)^{-1} \mathbf{V}_{1}^{\mathrm{H}} \mathbf{D}_{1}$ such that

$$
\left(\rho_{j} \tilde{P}\left(\mathrm{e}^{\mathrm{i} \omega_{j}}\right)\right)_{j=-M}^{M}=\tilde{\sigma} \mathbf{L}_{1} \tilde{\mathbf{v}}-\mathbf{L}_{1} \mathbf{E} \tilde{\mathbf{u}}
$$

and hence

$$
\rho_{0}^{2} \tilde{P}(1)^{2}+2 \sum_{j=1}^{M}\left|\rho_{j}\right|^{2}\left|\tilde{P}\left(\mathrm{e}^{\mathrm{i} \omega_{j}}\right)\right|^{2} \leq\left(|\tilde{\sigma}|+\|\mathbf{E}\|_{2}\right)^{2}\left\|\mathbf{L}_{1}\right\|_{2}^{2} .
$$

This completes the proof.

Lemma 4.5 If the assumptions of Theorem 4.4 are fulfilled with sufficiently small accuracies $\varepsilon_{1}, \varepsilon_{2}>0$ and if $\delta>0$ is the smallest singular value $\neq 0$ of $\mathbf{H}$, then

$$
\|\tilde{\mathbf{u}}-\mathbf{P} \tilde{\mathbf{u}}\| \leq \frac{\varepsilon_{2}+(N+1) \varepsilon_{1}}{\delta}
$$

where $\mathbf{P}$ is the orthogonal projector of $\mathbb{R}^{L+1}$ onto ker $\mathbf{H}$. Furthermore the polynomial (3.3) has zeros close to $\mathrm{e}^{\mathrm{i} \omega_{j}}(j=$ $-M, \ldots, M)$, where

$$
\begin{aligned}
& \tilde{P}(1)^{2}+2 \sum_{j=1}^{M}\left|\tilde{P}\left(\mathrm{e}^{\mathrm{i} \omega_{j}}\right)\right|^{2} \\
& \leq\left(2 N-L+1+\frac{2 \pi}{q}\left(1+\ln \frac{\pi}{q}\right)\right)\left(\frac{\varepsilon_{2}+(N+1) \varepsilon_{1}}{\delta}\right)^{2} .
\end{aligned}
$$

Proof. 1. Let $\tilde{\mathbf{u}}$ and $\tilde{\mathbf{v}}$ be normed right and left singular vectors of $\tilde{\mathbf{H}}$ with respect to the singular value $\tilde{\sigma} \in\left(0, \varepsilon_{2}\right]$ such that $\tilde{\mathbf{H}} \tilde{\mathbf{u}}=\tilde{\sigma} \tilde{\mathbf{v}}$. By assumption, $\delta>0$ is the smallest singular value $\neq 0$ of the exact Hankel matrix $\mathbf{H}$. Then $\delta^{2}$ is the smallest eigenvalue $\neq 0$ of the symmetric matrix $\mathbf{H}^{\mathrm{T}} \mathbf{H}$. Using the Rayleigh-Ritz Theorem (see [8, pp. 176-178]), we receive

$$
\delta^{2}=\min _{\substack{\mathbf{u} \neq \mathbf{0} \\ \mathbf{u} \perp \text { ker } \mathbf{H}}} \frac{\mathbf{u}^{\mathrm{T}} \mathbf{H}^{\mathrm{T}} \mathbf{H} \mathbf{u}}{\mathbf{u}^{\mathrm{T}} \mathbf{u}}
$$

and hence

$$
\delta=\min _{\substack{\mathbf{u} \neq 0 \\ \mathbf{u} \perp \text { ker } \mathbf{H}}} \frac{\|\mathbf{H u}\|}{\|\mathbf{u}\|}=\min _{\substack{\tilde{\tilde{u}} \mathbf{-} \neq \mathbf{0} \\ \tilde{\mathbf{u}} \mathbf{u} \mathbf{u} \mathbf{k} \mathbf{H} \mathbf{H}}} \frac{\|\mathbf{H}(\tilde{\mathbf{u}}-\mathbf{u})\|}{\|\tilde{\mathbf{u}}-\mathbf{u}\|} .
$$

Thus the following estimate

$$
\delta\|\tilde{\mathbf{u}}-\mathbf{u}\| \leq\|\mathbf{H}(\tilde{\mathbf{u}}-\mathbf{u})\|
$$

is true for all $\mathbf{u} \in \mathbb{R}^{L+1}$ with $\tilde{\mathbf{u}}-\mathbf{u} \perp \operatorname{ker} \mathbf{H}$. Especially for $\mathbf{u}=\mathbf{P} \tilde{\mathbf{u}}$, we see that $\tilde{\mathbf{u}}-\mathbf{P} \tilde{\mathbf{u}} \perp \operatorname{ker} \mathbf{H}$ and hence by (3.1)

$\delta\|\tilde{\mathbf{u}}-\mathbf{P} \tilde{\mathbf{u}}\| \leq\|\mathbf{H} \tilde{\mathbf{u}}\|=\|(\tilde{\mathbf{H}}-\mathbf{E}) \tilde{\mathbf{u}}\|=\|\tilde{\sigma} \tilde{\mathbf{v}}-\mathbf{E} \tilde{\mathbf{u}}\| \leq \tilde{\sigma}+(N+1) \varepsilon_{1}$ such that (4.9) follows.

2. Thereby $\mathbf{u}=\mathbf{P} \tilde{\mathbf{u}}$ is a right singular vector of $\mathbf{H}$ with respect to the singular value 0 . Thus the corresponding polynomial (2.5) has the values $\mathrm{e}^{\mathrm{i} \omega_{j}}(j=-M, \ldots, M)$ as zeros by Lemma 
2.2. By (4.9), the coefficients of $P$ differ only a little from the coefficients of $\tilde{P}$. Consequently, the zeros of $\tilde{P}$ lie nearby the zeros of $P$, i.e., $\tilde{P}$ has zeros close to $\mathrm{e}^{\mathrm{i} \omega_{j}}(j=-M, \ldots, M)$ (see [8, pp. 539-540]).

By $\left\|\mathbf{V}_{1}\right\|_{2}=\left\|\mathbf{V}_{1}^{\mathrm{H}}\right\|_{2},(4.9)$, and Corollary 4.3, we obtain the estimate

$$
\begin{aligned}
& \sum_{j=-M}^{M}\left|\tilde{P}\left(\mathrm{e}^{\mathrm{i} \omega_{j}}\right)\right|^{2}=\sum_{j=-M}^{M}\left|P\left(\mathrm{e}^{\mathrm{i} \omega_{j}}\right)-\tilde{P}\left(\mathrm{e}^{\mathrm{i} \omega_{j}}\right)\right|^{2} \\
= & \left\|\mathbf{V}_{1}^{\mathrm{H}}(\mathbf{u}-\tilde{\mathbf{u}})\right\|^{2} \leq\left\|\mathbf{V}_{1}^{\mathrm{H}}\right\|_{2}^{2}\|\mathbf{u}-\tilde{\mathbf{u}}\|^{2} \\
\leq & \left(2 N-L+1+\frac{2 \pi}{q}\left(1+\ln \frac{\pi}{q}\right)\right)\left(\frac{\varepsilon_{2}+(N+1) \varepsilon_{1}}{\delta}\right)^{2} .
\end{aligned}
$$

This completes the proof.

Corollary 4.6 Let $L, M \in \mathbb{N}$ with $L \geq 2 M+1$ be given, where $L$ is even. Furthermore let $\omega_{0}=0<\omega_{1}<\ldots<\omega_{M}<\pi$ with separation distance $q$ be given. Let (1.2) be fulfilled. Further let $N \in \mathbb{N}$ with

$$
N>\max \left\{\frac{\pi^{2}}{q}+\frac{L}{2}, L\right\}
$$

be given. Assume that the perturbed rectangular Hankel matrix (3.2) has a singular value $\tilde{\sigma} \in\left(0, \varepsilon_{2}\right]$ with corresponding normed right and left singular vectors $\tilde{\mathbf{u}}=\left(\tilde{u}_{n}\right)_{n=0}^{L} \in \mathbb{R}^{L+1}$ and $\tilde{\mathbf{v}}=\left(\tilde{v}_{n}\right)_{n=0}^{2 N-L+1} \in \mathbb{R}^{2 N-L+1}$.

Then the values $\left|\tilde{P}\left(\mathrm{e}^{\mathrm{i} \omega_{j}}\right)\right|(j=-M, \ldots, M)$ of (3.3) can be estimated by

$\rho_{0}^{2} \tilde{P}(1)^{2}+2 \sum_{j=1}^{M}\left|\rho_{j}\right|^{2}\left|\tilde{P}\left(\mathrm{e}^{\mathrm{i} \omega_{j}}\right)\right|^{2} \leq \frac{3}{2 N-L+2}\left(\varepsilon_{2}+(N+1) \varepsilon_{1}\right)^{2}$,

i.e., the values $\left|\tilde{P}\left(\mathrm{e}^{\mathrm{i} \omega_{j}}\right)\right|(j=-M, \ldots, M)$ are small. Further the polynomial $\tilde{P}$ has zeros close to $\mathrm{e}^{\mathrm{i} \omega_{j}}(j=-M, \ldots, M)$.

Proof. The assertion follows immediately by the assumption on $N$, the estimate (3.1), Corollary 4.2, and Theorem 4.4.

Now we can formulate a second approximate Prony method.

\section{Algorithm 4.7 (APM 2)}

Input: $L, N \in \mathbb{N}(3 \leq L \leq N, L$ is an upper bound of the number of exponentials), $\tilde{h}_{k}=f(k)+e_{k}(k=0, \ldots, 2 N)$ with $\left|e_{k}\right| \leq \varepsilon_{1}$, accuracies $\varepsilon_{1}, \varepsilon_{2}, \varepsilon_{3}, \varepsilon_{4}$.

1. Compute a right singular vector $\tilde{\mathbf{u}}^{(1)}=\left(\tilde{u}_{l}^{(1)}\right)_{l=0}^{L}$ corresponding to a singular value $\sigma^{(1)} \in\left(0, \varepsilon_{2}\right]$ of the perturbed rectangular Hankel matrix (3.2).

2. Form the corresponding polynomial $\tilde{P}^{(1)}(z):=\sum_{k=0}^{L} \tilde{u}_{k}^{(1)} z^{k}$ and evaluate all zeros $r_{j}^{(1)} \mathrm{e}^{\mathrm{i} \omega_{j}^{(1)}}\left(j=1, \ldots, M^{(1)}\right)$ with $\omega_{j}^{(1)} \in$ $(0, \pi),(1.2)$ and $\left|r_{j}^{(1)}-1\right| \leq \varepsilon_{4}$. Note that $L \geq 2 M^{(1)}+1$.

3. Compute a right singular vector $\tilde{\mathbf{u}}^{(2)}=\left(\tilde{u}_{l}^{(2)}\right)_{l=0}^{L}$ corresponding to a singular value $\sigma^{(2)} \in\left(0, \varepsilon_{2}\right]\left(\sigma^{(1)} \neq \sigma^{(2)}\right)$ of the perturbed rectangular Hankel matrix (3.2).

4. Form the corresponding polynomial $\tilde{P}^{(2)}(z):=\sum_{k=0}^{L} \tilde{u}_{k}^{(2)} z^{k}$ and evaluate all zeros $r_{k}^{(2)} \mathrm{e}^{\mathrm{i} \omega_{k}^{(2)}}\left(k=1, \ldots, M^{(2)}\right)$ with $\omega_{k}^{(2)} \in$
$(0, \pi),(1.2)$ and $\left|r_{k}^{(2)}-1\right| \leq \varepsilon_{4}$. Note that $L \geq 2 M^{(2)}+1$.

5. Determine all frequencies

$$
\tilde{\omega}_{l}:=\frac{1}{2}\left(\omega_{j(l)}^{(1)}+\omega_{k(l)}^{(2)}\right) \quad(l=1 \ldots, \tilde{M}),
$$

if there exist indices $j(l) \in\left\{1, \ldots, M^{(1)}\right\}$ and $k(l) \in\left\{1, \ldots, M^{(2)}\right\}$ such that $\left|\omega_{j(l)}^{(1)}-\omega_{k(l)}^{(2)}\right| \leq \varepsilon_{3}$. Replace $r_{j(l)}^{(1)}$ and $r_{k(l)}^{(2)}$ by 1 . Note that $L \geq 2 \tilde{M}+1$.

6. Compute $\tilde{\rho}_{0} \in \mathbb{R}$ and $\tilde{\rho}_{j} \in \mathbb{C}(j=1, \ldots, \tilde{M})$ with (1.2) as least squares solution of the overdetermined linear Vandermonde-type system

$$
\sum_{j=-\tilde{M}}^{\tilde{M}} \tilde{\rho}_{j} \mathrm{e}^{\mathrm{i} \tilde{\omega}_{j} k}=\tilde{h}_{k} \quad(k=0, \ldots, 2 N)
$$

with the diagonal preconditioner $\mathbf{D}=\operatorname{diag}(1-|k| /(N+1))_{k=-N}^{N}$. For very large $M$ and $N$ use the CGNR method (conjugate gradient on the normal equations, see e.g. [12, p. 288]), where the multiplication of the Vandermonde-type matrix $\tilde{\mathbf{V}}:=\left(\mathrm{e}^{\mathrm{i} k \tilde{\omega}_{j}}\right)_{k=0, j=-\tilde{M}}^{2 N, \tilde{M}}$ is realized in each iteration step by the nonequispaced fast Fourier transform (NFFT, see [9, 10].

7. Delete all the pairs $\left(\tilde{\omega}_{l}, \tilde{\rho}_{j}\right)(l \in\{1, \ldots, \tilde{M}\})$ with $\left|\tilde{\rho}_{l}\right| \leq \varepsilon_{1}$ and denote the remaining frequency set by $\left\{\omega_{j}: j=1, \ldots, M\right\}$ with $M \leq \tilde{M}$.

8. Repeat step 6 and solve the overdetermined linear Vandermonde-type system $\sum_{j=-M}^{M} \rho_{j} \mathrm{e}^{\mathrm{i} \omega_{j} k}=\tilde{h}_{k} \quad(k=0, \ldots, 2 N)$ with respect to the new frequency set $\left\{\omega_{j}: j=1, \ldots, M\right\}$ again.

Output: $M \in \mathbb{N}, \rho_{0} \in \mathbb{R}, \rho_{j} \in \mathbb{C}\left(\rho_{j}=\bar{\rho}_{-j}\right), \omega_{j} \in(0, \pi)$ $(j=1, \ldots, M)$.

We can determine the zeros of the polynomials $\tilde{P}^{(1)}$ and $\tilde{P}^{(2)}$ by computing the eigenvalues of the related companion matrices, see Remark 2.4. Note that by Corollary 4.6, the correct frequencies $\omega_{j}$ can be approximately computed by any right singular vector corresponding to a small singular value of (3.2). In general, the additional roots are not nearby for different right singular vectors. In many applications, Algorithm 4.7 can be essentially simplified by using only one right singular vector (cf. steps $1-2$ ), omitting the steps $3-5$, and determining the frequencies, the coefficients and the number $M$ in the steps $6-$ 8. We use this simplified variant of Algorithm 4.7 in our numerical examples of Section 6.

Note that more advanced methods for computing the unknown frequencies were developed, such as matrix pencil methods. In $[13,14]$ a relationship between the matrix pencil methods and several variants of the ESPRIT method $[15,16]$ is derived showing comparable performance. Now we replace the steps $1-5$ of Algorithm 4.7 by the least squares ESPRIT method [17, p. 493]. This leads to the following

\section{Algorithm 4.8 (APM based on ESPRIT)}

Input: $L, N, P \in \mathbb{N}(3 \leq L \leq N, L$ is an upper bound of the number of exponentials, $P+1$ is upper bound of the number of positive frequencies, $P+1 \leq L), \tilde{h}_{k}=f(k)+e_{k}(k=0, \ldots, 2 N)$ 
with $\left|e_{k}\right| \leq \varepsilon_{1}$.

1. Form the perturbed rectangular Hankel matrix (3.2).

2. Compute the singular value decomposition of (3.2) with a diagonal matrix $\mathbf{S} \in \mathbb{R}^{(2 N-L+1) \times(L+1)}$ with nonnegative diagonal elements in decreasing order, and unitary matrices $\mathbf{L} \in$ $\mathbb{C}^{(2 N-L+1) \times(2 N-L+1)}$ and $\mathbf{U}:=\left(u_{k, l}\right)_{k, l=0}^{L} \in \mathbb{C}^{(L+1) \times(L+1)}$ such that $\tilde{\mathbf{H}}=\mathbf{L} \mathbf{S} \mathbf{U}^{\mathrm{H}}$.

3. Form the matrices $\mathbf{U}_{1}:=\left(u_{k, l}\right)_{k, l=0}^{L-1, P}$ and $\mathbf{U}_{2}:=\left(u_{k+1, l}\right)_{k, l=0}^{L-1, P}$.

4. Compute the matrix $\mathbf{P}:=\mathbf{U}_{1}^{\dagger} \mathbf{U}_{2} \in \mathbb{C}^{(P+1) \times(P+1)}$, where $\mathbf{U}_{1}^{\dagger}:=\left(\mathbf{U}_{1}^{\mathrm{H}} \mathbf{U}_{1}\right)^{-1} \mathbf{U}_{1}^{\mathrm{H}}$ is the Moore-Penrose pseudoinverse of $\mathbf{U}_{1}$. 5. Compute the eigenvalues $\tilde{r}_{k} \mathrm{e}^{\mathrm{i} \tilde{\omega}_{k}}(k=1, \ldots, P+1)$ of the matrix $\mathbf{P}$, where $\tilde{\omega}_{k} \in(0, \pi)$ and $\tilde{r}_{k} \approx 1(k=1, \ldots, P+1)$.

6. Compute $\tilde{\rho}_{0} \in \mathbb{R}$ and $\tilde{\rho}_{j} \in \mathbb{C}(j=1, \ldots, P+1)$ with (1.2) as least squares solution of the overdetermined linear Vandermonde-type system

$$
\sum_{j=-P-1}^{P+1} \tilde{\rho}_{j} \mathrm{e}^{\mathrm{i} \tilde{\omega}_{j} k}=\tilde{h}_{k} \quad(k=0, \ldots, 2 N)
$$

with the diagonal preconditioner $\mathbf{D}=\operatorname{diag}(1-|k| /(N+1))_{k=-N}^{N}$. For large $P$ and $N$, use the CGNR method, where the multiplication of the Vandermonde-type matrix $\left(\mathrm{e}^{\mathrm{i} k \tilde{\omega}_{j}}\right)_{k=0, j=-P-1}^{2 N, P+1}$ is realized in each iteration step by NFFT (see $[9,10])$.

7. Delete all the pairs $\left(\tilde{\omega}_{l}, \tilde{\rho}_{j}\right)(l \in\{1, \ldots, P+1\})$ with $\left|\tilde{\rho}_{l}\right| \leq \varepsilon_{1}$ and denote the remaining frequency set by $\left\{\omega_{j}: j=1, \ldots, M\right\}$ with $M \leq P+1$.

8. Repeat step 6 and solve the overdetermined linear Vandermonde-type system $\sum_{j=-M}^{M} \rho_{j} \mathrm{e}^{\mathrm{i} \omega_{j} k}=\tilde{h}_{k} \quad(k=$ $0, \ldots, 2 N)$ with respect to the new frequency set $\left\{\omega_{j}: j=1, \ldots, M\right\}$ again.

Output: $M \in \mathbb{N}, \rho_{0} \in \mathbb{R}, \rho_{j} \in \mathbb{C}\left(\rho_{j}=\bar{\rho}_{-j}\right), \omega_{j} \in(0, \pi)$ $(j=1, \ldots, M)$.

Since a good estimate for the number of positive frequencies is often unknown in advance, we can simply choose $P=L-1$ in our numerical experiments. Clearly, we can choose the parameter $L$ similarly as suggested in [14] based on the accuracy $\varepsilon_{2}$ as in Algorithm 4.7. Furthermore we would like to point out that in Algorithm 4.8 the right singular vectors of $\tilde{\mathbf{H}}$ related to the small singular values are discarded, otherwise the Algorithm 4.7 uses right singular vectors related to the small singular values in order to compute the roots of the corresponding polynomials.

Note that Algorithm 4.8 requires more arithmetical operations than Algorithm 4.7, due to the evaluation of the Moore-Penrose pseudoinverse of $\mathbf{U}_{1}$ in step 4 of Algorithm 4.8.

\section{Sensitivity analysis of the approximate Prony method}

In this section, we discuss the sensitivity of step 6 of the Algorithms 4.7 and 4.8, respectively. Assume that $N \geq 2 M+1$ and $\tilde{M}=M$. We solve the overdetermined linear Vandermonde- type system (4.10) with $M=\tilde{M}$ as weighted least squares problem

$$
\left\|\mathbf{D}^{1 / 2}(\tilde{\mathbf{V}} \tilde{\boldsymbol{\rho}}-\tilde{\mathbf{h}})\right\|=\min .
$$

Here $\tilde{\mathbf{h}}=\left(\tilde{h}_{k}\right)_{k=0}^{2 N}$ is the perturbed data vector and

$$
\tilde{\mathbf{V}}=\left(\mathrm{e}^{\mathrm{i} k \tilde{\omega}_{j}}\right)_{k=0, j=-M}^{2 N, M}
$$

is the Vandermonde-type matrix with the computed frequencies $\tilde{\omega}_{j}(j=-M, \ldots, M)$, where $0=\tilde{\omega}_{0}<\tilde{\omega}_{1}<\ldots<\tilde{\omega}_{M}<\pi$, $\tilde{\omega}_{j}=-\tilde{\omega}_{-j}(j=-M, \ldots,-1)$ and $\tilde{q}$ is the separation distance of the computed frequency set $\left\{\tilde{\omega}_{j}: j=0, \ldots, M+1\right\}$ with $\tilde{\omega}_{M+1}=2 \pi-\tilde{\omega}_{M}$. Note that $\tilde{\mathbf{V}}$ has full rank and that the unique solution of (5.1) is given by $\tilde{\boldsymbol{\rho}}=\tilde{\mathbf{L}} \tilde{\mathbf{h}}$ with the left inverse $\tilde{\mathbf{L}}=$ $\left(\tilde{\mathbf{V}}^{\mathrm{H}} \mathbf{D} \tilde{\mathbf{V}}\right)^{-1} \tilde{\mathbf{V}}^{\mathrm{H}} \mathbf{D}$ of $\tilde{\mathbf{V}}$.

We begin with a normwise perturbation result, if all frequencies are exactly determined, i.e., $\omega_{j}=\tilde{\omega}_{j}(j=-M, \ldots, M)$, and if a perturbed data vector $\tilde{\mathbf{h}}$ is given.

Lemma 5.1 Assume that $\omega_{j}=\tilde{\omega}_{j}(j=-M, \ldots, M)$ and that $\left|\tilde{h}_{k}-h_{k}\right| \leq \varepsilon_{1}$. Let $\mathbf{V}$ be given by (4.1). Further let $\mathbf{V} \boldsymbol{\rho}=$ $\mathbf{h} \neq \mathbf{0}$ and $\tilde{\boldsymbol{\rho}}=\mathbf{L} \tilde{\mathbf{h}}$, where (4.3) is a left inverse of $\mathbf{V}$. If the assumptions of Corollary 4.2 are fulfilled, then for each $N \in \mathbb{N}$ with $N>\pi^{2} q^{-1}$ the condition number $\kappa(\mathbf{V}):=\|\mathbf{L}\|_{2}\|\mathbf{V}\|_{2}$ is uniformly (with respect to $N$ ) bounded by

$$
\kappa(\mathbf{V}) \leq \sqrt{3+\frac{3}{\pi}\left(1+\ln \frac{\pi}{q}\right)} .
$$

Furthermore, the following stability inequalities are fulfilled

$$
\begin{aligned}
&\|\boldsymbol{\rho}-\tilde{\boldsymbol{\rho}}\| \leq \sqrt{\frac{3}{2 N+2}}\|\mathbf{h}-\tilde{\mathbf{h}}\| \leq \sqrt{3} \varepsilon_{1}, \\
& \frac{\|\boldsymbol{\rho}-\tilde{\boldsymbol{\rho}}\|}{\|\boldsymbol{\rho}\|} \leq \kappa(\mathbf{V}) \frac{\|\mathbf{h}-\tilde{\mathbf{h}}\|}{\|\mathbf{h}\|} .
\end{aligned}
$$

Proof. 1. The condition number $\kappa(\mathbf{V})$ of the rectangular Vandermonde-type matrix $\mathbf{V}$ is defined as the number $\|\mathbf{L}\|_{2}\|\mathbf{V}\|_{2}$. Note that $\kappa(\mathbf{V})$ does not coincide with the condition number of $\mathbf{V}$ related to the spectral norm, since the left inverse $\mathbf{L}$ is not the Moore-Penrose pseudoinverse of $\mathbf{V}$ by $\mathbf{D} \neq \mathbf{I}$. Applying the Corollaries 4.2 and 4.3 , we receive that

$$
\kappa(\mathbf{V}) \leq \sqrt{3+\frac{3 \pi}{(N+1) q}\left(1+\ln \frac{\pi}{q}\right)} .
$$

This provides (5.2), since by assumption $(N+1) q>\pi^{2}$.

2. The inequality (5.3) follows immediately from $\|\rho-\tilde{\rho}\| \leq$ $\|\mathbf{L}\|_{2}\|\mathbf{h}-\tilde{\mathbf{h}}\|$ and Corollary 4.2. The estimate (5.4) arises from (5.3) by multiplication with $\|\mathbf{V} \rho\|\|\mathbf{h}\|^{-1}=1$.

Now we consider the general case, where the weighted least squares problem (5.1) is solved for perturbed data $\tilde{h}_{k}(k=$ $0, \ldots, 2 N)$ and computed frequencies $\tilde{\omega}_{j}(j=-M, \ldots, M)$.

Theorem 5.2 Assume that $\left|\tilde{h}_{k}-h_{k}\right| \leq \varepsilon_{1}(k=0, \ldots, 2 N)$ and $\left|\tilde{\omega}_{j}-\omega_{j}\right| \leq \delta(j=0, \ldots, M)$. Let $\mathbf{V}$ be given by (4.1) and let

$$
\tilde{\mathbf{V}}:=\left(\mathrm{e}^{\mathrm{i} k \tilde{\omega}_{j}}\right)_{k=0, j=-M}^{2 N, M} .
$$


Further let $\mathbf{V} \boldsymbol{\rho}=\mathbf{h}$ and $\tilde{\boldsymbol{\rho}}=\tilde{\mathbf{L}} \tilde{\mathbf{h}}$, where $\tilde{\mathbf{L}}=\left(\tilde{\mathbf{V}}^{\mathrm{H}} \mathbf{D} \tilde{\mathbf{V}}\right)^{-1} \tilde{\mathbf{V}}^{\mathrm{H}} \mathbf{D}$ is a left inverse of $\tilde{\mathbf{V}}$. If the assumptions of Corollary 4.2 are fulfilled, then for each $N \in \mathbb{N}$ with

$$
N>\pi^{2} \max \left\{q^{-1}, \tilde{q}^{-1}\right\}
$$

the following estimate

$$
\|\boldsymbol{\rho}-\tilde{\boldsymbol{\rho}}\| \leq \sqrt{(6 N+6)(2 M+1)}\|\mathbf{h}\| \delta+\sqrt{3} \varepsilon_{1}
$$

is fulfilled.

Proof. 1. Using the matrix-vector notation, we can write (2.6) in the form $\mathbf{V} \boldsymbol{\rho}=\mathbf{h}$. The overdetermined linear system (4.10) with $\tilde{M}=M$ reads $\tilde{\mathbf{V}} \tilde{\boldsymbol{\rho}}=\tilde{\mathbf{h}}$. Using the left inverse $\tilde{\mathbf{V}}$, the solution $\tilde{\boldsymbol{\rho}}$ of the weighted least squares problem

$$
\left\|\mathbf{D}^{1 / 2}(\tilde{\mathbf{V}} \tilde{\boldsymbol{\rho}}-\tilde{\mathbf{h}})\right\|=\min
$$

is $\tilde{\boldsymbol{\rho}}=\tilde{\mathbf{L}} \tilde{\mathbf{h}}$. Thus it follows that

$$
\begin{aligned}
\|\boldsymbol{\rho}-\tilde{\boldsymbol{\rho}}\| & =\|\boldsymbol{\rho}-\tilde{\mathbf{L}} \tilde{\mathbf{h}}\| \\
& =\|\tilde{\mathbf{L}} \tilde{\mathbf{V}} \boldsymbol{\rho}-\tilde{\mathbf{L}} \mathbf{V} \boldsymbol{\rho}-\tilde{\mathbf{L}}(\tilde{\mathbf{h}}-\mathbf{h})\| \\
& \leq\|\tilde{\mathbf{L}}\|_{2}\|\tilde{\mathbf{V}}-\mathbf{V}\|_{2}\|\boldsymbol{\rho}\|+\|\tilde{\mathbf{L}}\|_{2}\|\tilde{\mathbf{h}}-\mathbf{h}\| .
\end{aligned}
$$

2. Now we estimate the squared spectral norm of $\tilde{\mathbf{V}}-\mathbf{V}$ by means of the Frobenius norm

$$
\begin{aligned}
& \|\mathbf{V}-\tilde{\mathbf{V}}\|_{2}^{2} \leq\|\mathbf{V}-\tilde{\mathbf{V}}\|_{\mathrm{F}}^{2}=\sum_{k=0}^{2 N} \sum_{j=-M}^{M}\left|\mathrm{e}^{\mathrm{i} k \omega_{j}}-\mathrm{e}^{\mathrm{i} k \tilde{\omega}_{j}}\right|^{2} \\
& =\sum_{j=-M}^{M}\left(\sum_{k=0}^{2 N}\left[2-2 \cos \left(\left(\omega_{j}-\tilde{\omega}_{j}\right) k\right)\right]\right) \\
& =\sum_{j=-M}^{M}\left(4 N+1-\frac{\sin \left((2 N+1 / 2)\left(\omega_{j}-\tilde{\omega}_{j}\right)\right)}{\left.\sin \left(\left(\omega_{j}-\tilde{\omega}_{j}\right) / 2\right)\right)}\right) .
\end{aligned}
$$

Using the special property of the Dirichlet kernel

$$
\frac{\sin (4 N+1) x / 2}{\sin x / 2} \geq 4 N+1+\left(-\frac{1}{3}\left(2 N+\frac{1}{2}\right)^{3}+\frac{N}{6}+\frac{1}{24}\right) x^{2},
$$

we infer

$$
\begin{aligned}
\|\mathbf{V}-\tilde{\mathbf{V}}\|_{2}^{2} & \leq \sum_{j=-M}^{M}\left(\frac{1}{3}\left(2 N+\frac{1}{2}\right)^{3}-\frac{N}{6}-\frac{1}{24}\right) \delta^{2} \\
& \leq \frac{(2 N+1 / 2)^{3}(2 M+1)}{3} \delta^{2} .
\end{aligned}
$$

Thus we receive

$$
\|\boldsymbol{\rho}-\tilde{\boldsymbol{\rho}}\| \leq \frac{1}{\sqrt{3}}\left(2 N+\frac{1}{2}\right)^{3 / 2} \sqrt{2 M+1}\|\tilde{\mathbf{L}}\|\|\boldsymbol{\rho}\| \delta+\|\tilde{\mathbf{L}}\|_{2}\|\tilde{\mathbf{h}}-\mathbf{h}\| .
$$

From Corollary 4.2 it follows that for each $N \in \mathbb{N}$ with (5.5)

$$
\|\tilde{\mathbf{L}}\|_{2} \leq \sqrt{\frac{3}{2 N+2}}
$$

Finally we use

$$
\begin{aligned}
\|\tilde{\mathbf{h}}-\mathbf{h}\| & \leq \sqrt{2 N+1}\|\tilde{\mathbf{h}}-\mathbf{h}\|_{\infty} \leq \sqrt{2 N+1} \varepsilon_{1}, \\
\|\rho\| & =\|\mathbf{L} \mathbf{h}\| \leq\|\mathbf{L}\|_{2}\|\mathbf{h}\| \leq \sqrt{\frac{3}{2 N+2}}\|\mathbf{h}\|
\end{aligned}
$$

and obtain the result.

By Theorem 5.2, we see that we have to compute the frequencies very carefully. This is the reason why we repeat the computation of the frequencies in the steps 3-4 of Algorithm 4.7.

\section{Numerical examples}

Finally, we apply the Algorithms 4.7 and 4.8 to various examples. We have implemented our algorithms in MATLAB with IEEE double precision arithmetic. There exist a variety of algorithms to recover the frequencies $\omega_{j}$ like ESPRIT $[15,16]$ and more advanced methods like total least squares phased averaging ESPRIT [18, 19]. We emphasize that the aim of this paper is not a widespread numerical comparison with all the existing methods. We demonstrate that a straightforward application of the ESPRIT method [17, p. 493] leads to similar results.

Let $\omega:=\left(\omega_{j}\right)_{j=0}^{M}$ and $\rho:=\left(\rho_{j}\right)_{j=0}^{M}$. We compute the frequency error $e(\omega):=\max _{j=0, \ldots, M}\left|\omega_{j}-\tilde{\omega}_{j}\right|$, where $\tilde{\omega}_{j}$ are computed by Algorithm 4.7 and 4.8, respectively. Furthermore let $e(\rho):=$ $\max _{j=0, \ldots, M}\left|\rho_{j}-\tilde{\rho}_{j}\right|$ be the error of the coefficients. Finally we determine the error $e(f):=\max |f(x)-\tilde{f}(x)| / \max |f(x)|$ computed on 10000 equidistant points of the interval $[0,2 N]$, where $\tilde{f}$ is the recovered exponential sum. For increasing number of sampled data, we obtain a very precise parameter estimation. With other words, $N$ acts as regularization parameter.

Example 6.1 We sample the trigonometric sum

$$
\begin{aligned}
& f_{1}(x):=14-8 \cos (0.453 x)+9 \sin (0.453 x) \\
& +4 \cos (0.979 x)+8 \sin (0.979 x)-2 \cos (0.981 x) \\
& +2 \cos (1.847 x)-3 \sin (1.847 x)+0.1 \cos (2.154 x) \\
& -0.3 \sin (2.154 x)
\end{aligned}
$$

at the equidistant nodes $x=k(k=0, \ldots, 2 N)$. Then we apply the Algorithms 4.7 and 4.8 with exact sampled data $h_{k}=f_{1}(k)$, i.e., $e_{k}=0(k=0, \ldots, 2 N)$. Thus the accuracy $\varepsilon_{1}$ can be chosen as the unit roundoff $\varepsilon_{1}=2^{-53} \approx 1.11 \times 10^{-16}$ (see [20, p. 45]). Note that the frequencies $\omega_{2}=0.979$ and $\omega_{3}=0.981$ are very close, so that $q=0.002$. Nevertheless for small $N$ and for the accuracies $\varepsilon_{2}=\varepsilon_{3}=\varepsilon_{4}=10^{-7}$, we obtain very precise results, see Table 6.1. Here we assume that $P$ is unknown and use the estimate $P=L-1$ in Algorithm 4.8 .

Example 6.2 We use again the function (6.1). Now we consider noisy sampled data $\tilde{h}_{k}=f_{1}(k)+e_{k}(k=0, \ldots, 2 N)$, where $e_{k}$ is a random error with $\left|e_{k}\right| \leq \varepsilon_{1}=5 \cdot 10^{-5}$, more precisely we add pseudorandom values drawn from the standard uniform distribution on $\left[-5 \cdot 10^{-5}, 5 \cdot 10^{-5}\right]$. Now we choose $\varepsilon_{4}<5 \cdot 10^{-5}$. We see that for noisy sampled data increasing $N$ improves the results, see Table 6.2. The last column shows the signal-tonoise ratio SNR := $10 \log _{10}\left(\left\|\left(f_{1}(k)\right)_{k=0}^{2 N}\right\| /\left\|\left(e_{k}\right)_{k=0}^{2 N}\right\|\right)$. 


\begin{tabular}{|c|c||c|c|c|}
\hline$N$ & $L$ & $e(\omega)$ & $e(\rho)$ & $e\left(f_{1}\right)$ \\
\hline \hline \multicolumn{5}{|c|}{ Algorithm 4.7 } \\
\hline 12 & 11 & $1.303 \mathrm{e}-09$ & $2.624 \mathrm{e}-06$ & $3.257 \mathrm{e}-14$ \\
\hline 20 & 11 & $2.057 \mathrm{e}-11$ & $5.527 \mathrm{e}-08$ & $1.703 \mathrm{e}-13$ \\
\hline 20 & 19 & $6.505 \mathrm{e}-12$ & $4.370 \mathrm{e}-13$ & $1.202 \mathrm{e}-14$ \\
\hline \hline \multicolumn{5}{|c|}{ Algorithm 4.8 } \\
\hline 12 & 11 & $6.971 \mathrm{e}-10$ & $1.390 \mathrm{e}-06$ & $5.383 \mathrm{e}-14$ \\
\hline 20 & 11 & $2.199 \mathrm{e}-11$ & $5.387 \mathrm{e}-08$ & $9.778 \mathrm{e}-14$ \\
\hline 20 & 19 & $6.926 \mathrm{e}-12$ & $9.948 \mathrm{e}-14$ & $1.457 \mathrm{e}-14$ \\
\hline
\end{tabular}

Table 6.1: Results for $f_{1}$ from Example 6.1.

\begin{tabular}{|c|c||c|c|c|c|}
\hline$N$ & $L$ & $e(\omega)$ & $e(\rho)$ & $e\left(f_{1}\right)$ & SNR \\
\hline \hline \multicolumn{7}{|c|}{ Algorithm 4.7} \\
\hline 20 & 11 & $9.876 \mathrm{e}-01$ & $3.992 \mathrm{e}-02$ & $1.338 \mathrm{e}-04$ & 52.64 \\
\hline 60 & 11 & $2.557 \mathrm{e}-02$ & $9.801 \mathrm{e}-04$ & $2.807 \mathrm{e}-04$ & 52.61 \\
\hline 60 & 19 & $1.098 \mathrm{e}-03$ & $1.017 \mathrm{e}-04$ & $1.771 \mathrm{e}-05$ & 52.53 \\
\hline 60 & 29 & $4.135 \mathrm{e}-04$ & $3.744 \mathrm{e}-06$ & $5.130 \mathrm{e}-06$ & 52.81 \\
\hline \hline \multicolumn{7}{|c|}{ Algorithm 4.8} \\
\hline 20 & 11 & $4.006 \mathrm{e}-01$ & $5.283 \mathrm{e}-04$ & $3.070 \mathrm{e}-05$ & 52.85 \\
\hline 60 & 11 & $5.197 \mathrm{e}-02$ & $4.762 \mathrm{e}-04$ & $3.589 \mathrm{e}-04$ & 52.60 \\
\hline 60 & 19 & $2.536 \mathrm{e}-04$ & $3.421 \mathrm{e}-05$ & $4.991 \mathrm{e}-06$ & 52.94 \\
\hline 60 & 29 & $5.493 \mathrm{e}-04$ & $2.362 \mathrm{e}-06$ & $6.440 \mathrm{e}-06$ & 52.54 \\
\hline
\end{tabular}

Table 6.2: Results for $f_{1}$ from Example 6.2

Example 6.3 As in [21], we sample the 24-periodic trigonometric sum

$f_{2}(x):=2 \cos \left(\frac{\pi x}{6}\right)+200 \cos \left(\frac{\pi x}{4}\right)+2 \cos \left(\frac{\pi x}{2}\right)+2 \cos \left(\frac{5 \pi x}{6}\right)$

at the equidistant nodes $x=k(k=0, \ldots, 2 N)$. In [21], only the dominant frequency $\omega_{2}=\pi / 4$ is iteratively computed via zeros of Szegö polynomials. For $N=50, \omega_{2}$ is determined to 2 correct decimal places. For $N=200$ and $N=500$, the frequency $\omega_{2}$ is computed to 4 correct decimal places in [21].

Now we apply the Algorithms 4.7 and 4.8 with the same parameters as in Example 6.1, but we use only the 19 sampled data $f_{2}(k)(k=0, \ldots, 18)$. In this case we are able to compute all frequencies and all coefficients very accurate, see Table 6.3.

Example 6.4 A different method for finding the frequencies based on detection of singularities is suggested in [22], where in addition this method is also suitable for more difficult problems, such as finding singularities of different orders and estimating the local Lipschitz exponents. Similarly as in [22], we consider the 8-periodic test function

$$
f_{3}(x):=34+300 \cos (\pi x / 4)+\cos (\pi x / 2)
$$

\begin{tabular}{|c|c||c|c|c|}
\hline$N$ & $L$ & $e(\omega)$ & $e(\rho)$ & $e\left(f_{2}\right)$ \\
\hline \hline \multicolumn{5}{|c|}{ Algorithm 4.7 } \\
\hline 9 & 8 & $4.996 \mathrm{e}-15$ & $1.037 \mathrm{e}-12$ & $7.123 \mathrm{e}-15$ \\
\hline 50 & 8 & $7.883 \mathrm{e}-15$ & $2.728 \mathrm{e}-12$ & $3.045 \mathrm{e}-14$ \\
\hline 50 & 49 & $9.548 \mathrm{e}-15$ & $1.536 \mathrm{e}-11$ & $1.664 \mathrm{e}-13$ \\
\hline \hline \multicolumn{5}{|c|}{ Algorithm 4.8 } \\
\hline 9 & 8 & $5.662 \mathrm{e}-15$ & $1.847 \mathrm{e}-13$ & $6.1500 \mathrm{e}-15$ \\
\hline 50 & 8 & $8.105 \mathrm{e}-15$ & $4.066 \mathrm{e}-13$ & $8.6300 \mathrm{e}-14$ \\
\hline 50 & 49 & $2.442 \mathrm{e}-15$ & $1.423 \mathrm{e}-11$ & $1.0765 \mathrm{e}-13$ \\
\hline
\end{tabular}

Table 6.3: Results for $f_{2}$ from Example 6.3.

and sample $f_{3}$ at the nodes $x=k(k=0, \ldots, 2 N)$. Note that in [22], uniform noise in the range [0,3] is added and this experiment is repeated 500 times. Then the frequencies are recovered very accurately. In this case, the noise exceeds the lowest coefficient of $f_{3}$.

Now we use Algorithm 4.7 and 4.8, where we add noise in the range $[0, R]$ to the sampled data $f_{3}(k)(k=0, \ldots, 2 N)$. For $R=1$ and $R=3$ we obtain that the SNR is approximately 28 and 24 , respectively.. Of course, we can compute the three frequencies by choosing $L=3$ and select all frequencies, i.e., we choose $\varepsilon_{1}=0$. However in this case the error $e(\rho)$ has a high oscillation depending on the noise, therefore we repeat the experiment also 50 times and present the averages of the errors in Table 6.4.

Example 6.5 Finally, we consider the function

$$
f_{4}(x):=\sum_{j=1}^{40} \cos \left(\omega_{j} x\right)
$$

where we choose random frequencies $\omega_{j}$ drawn from the standard uniform distribution on $(0, \pi)$. We sample the function $f_{4}$ at the equidistant nodes $x=k(k=0, \ldots, 2 N)$. For the results of the Algorithms 4.7 and 4.8 see Table 6.5. Note that without the diagonal preconditioner $\mathbf{D}$ the overdetermined linear Vandermonde-type system is not numerically solvable due to the ill-conditioning of the Vandermonde-type matrix.

In summary, we obtain very accurate results already for relatively few sampled data. We can analyze both periodic and nonperiodic functions without preprocessing (as filtering or windowing). APM works correctly for noisy sampled data and for clustered frequencies assumed that the separation distance of the frequencies is not too small and the number $2 N+1$ of sampled data is sufficiently large. We can also use the ESPRIT method in order to determine the frequencies. Overmodeling improves the results. The numerical examples show that one can choose the number $2 N+1$ of sampled data less than expected by the theoretical results. We have essentially improved the stability of our APM by using a weighted least squares method. Our numerical examples confirm that the proposed APM is robust with respect to noise. 


\begin{tabular}{|c|c|c||c|c|c|}
\hline$N$ & $L$ & $R$ & $e(\omega)$ & $e(\rho)$ & $e\left(f_{3}\right)$ \\
\hline \hline \multicolumn{7}{|c|}{ Algorithm 4.7} \\
\hline 32 & 5 & 1 & $4.718 \mathrm{e}-03$ & $6.263 \mathrm{e}-01$ & $3.468 \mathrm{e}-03$ \\
\hline 64 & 5 & 1 & $3.438 \mathrm{e}-03$ & $6.028 \mathrm{e}-01$ & $3.448 \mathrm{e}-03$ \\
\hline 128 & 5 & 1 & $1.672 \mathrm{e}-03$ & $6.686 \mathrm{e}-01$ & $3.595 \mathrm{e}-03$ \\
\hline 256 & 5 & 1 & $1.205 \mathrm{e}-03$ & $6.134 \mathrm{e}-01$ & $3.901 \mathrm{e}-03$ \\
\hline 512 & 5 & 1 & $1.192 \mathrm{e}-03$ & $6.512 \mathrm{e}-01$ & $4.519 \mathrm{e}-03$ \\
\hline 1024 & 5 & 1 & $6.521 \mathrm{e}-04$ & $6.505 \mathrm{e}-01$ & $4.601 \mathrm{e}-03$ \\
\hline \hline 32 & 5 & 3 & $4.336 \mathrm{e}-02$ & $1.716 \mathrm{e}+00$ & $9.667 \mathrm{e}-03$ \\
\hline 64 & 5 & 3 & $2.257 \mathrm{e}-02$ & $1.969 \mathrm{e}+00$ & $1.037 \mathrm{e}-02$ \\
\hline 128 & 5 & 3 & $1.711 \mathrm{e}-02$ & $1.405 \mathrm{e}+00$ & $1.058 \mathrm{e}-02$ \\
\hline 256 & 5 & 3 & $1.089 \mathrm{e}-02$ & $1.503 \mathrm{e}+00$ & $1.154 \mathrm{e}-02$ \\
\hline 512 & 5 & 3 & $7.551 \mathrm{e}-03$ & $1.487 \mathrm{e}+00$ & $1.011 \mathrm{e}-02$ \\
\hline 1024 & 5 & 3 & $6.261 \mathrm{e}-03$ & $1.710 \mathrm{e}+00$ & $1.118 \mathrm{e}-02$ \\
\hline \hline \multicolumn{7}{|c|}{} \\
\hline 32 & 5 & 1 & $3.992 \mathrm{e}-03$ & $5.531 \mathrm{e}-01$ & $3.348 \mathrm{e}-03$ \\
\hline 64 & 5 & 1 & $2.953 \mathrm{e}-03$ & $6.538 \mathrm{e}-01$ & $3.501 \mathrm{e}-03$ \\
\hline 128 & 5 & 1 & $2.068 \mathrm{e}-03$ & $5.835 \mathrm{e}-01$ & $3.537 \mathrm{e}-03$ \\
\hline 256 & 5 & 1 & $1.329 \mathrm{e}-03$ & $6.833 \mathrm{e}-01$ & $3.818 \mathrm{e}-03$ \\
\hline 512 & 5 & 1 & $8.220 \mathrm{e}-04$ & $7.226 \mathrm{e}-01$ & $4.140 \mathrm{e}-03$ \\
\hline 1024 & 5 & 1 & $7.259 \mathrm{e}-04$ & $5.172 \mathrm{e}-01$ & $4.543 \mathrm{e}-03$ \\
\hline \hline 32 & 5 & 3 & $3.849 \mathrm{e}-02$ & $1.618 \mathrm{e}+00$ & $1.047 \mathrm{e}-02$ \\
\hline 64 & 5 & 3 & $2.254 \mathrm{e}-02$ & $1.835 \mathrm{e}+00$ & $1.054 \mathrm{e}-02$ \\
\hline 128 & 5 & 3 & $1.745 \mathrm{e}-02$ & $1.680 \mathrm{e}+00$ & $1.073 \mathrm{e}-02$ \\
\hline 256 & 5 & 3 & $1.152 \mathrm{e}-02$ & $2.025 \mathrm{e}+00$ & $1.091 \mathrm{e}-02$ \\
\hline 512 & 5 & 3 & $9.114 \mathrm{e}-03$ & $2.159 \mathrm{e}+00$ & $1.158 \mathrm{e}-02$ \\
\hline 1024 & 5 & 3 & $7.110 \mathrm{e}-03$ & $1.866 \mathrm{e}+00$ & $1.102 \mathrm{e}-02$ \\
\hline
\end{tabular}

Table 6.4: Results for $f_{3}$ from Example 6.4.

\section{Acknowledgment}

The first named author gratefully acknowledges the support by the German Research Foundation within the project KU 2557/1-1. Moreover, the authors would like to thank the referees for their valuable suggestions.

\section{References}

[1] G. Beylkin, L. Monzón, On approximations of functions by exponential sums, Appl. Comput. Harmon. Anal. 19 (2005) 17 - 48.

[2] W. L. Briggs, V. E. Henson, The DFT, SIAM, Philadelphia, 1995.

[3] J. M. Papy, L. De Lathauwer, S. Van Huffel, Exponential data fitting using multilinear algebra: the single-channel and multi-channel case, Numer. Linear Algebra Appl. 12 (2005) 809 - 826.

[4] D. Potts, M. Tasche, Numerical stability of nonequispaced fast Fourier transforms, J. Comput. Appl. Math 222 (2008) 655 - 674.

[5] S. L. Marple, Jr., Digital spectral analysis with applications, Prentice Hall Signal Processing Series, Prentice Hall Inc., Englewood Cliffs, 1987.

[6] G. H. Golub, P. Milanfar, J. Varah, A stable numerical method for inverting shape from moments, SIAM J. Sci. Comput. 21 (1999) 1222 - 1243.

[7] P. L. Dragotti, M. Vetterli, T. Blu, Sampling moments and reconstructing signals of finite rate of innovation: Shannon meets Strang-Fix, IEEE Trans. Signal Process. 55 (2007) 1741 - 1757.

[8] R. A. Horn, C. R. Johnson, Matrix Analysis, Cambridge University Press, Cambridge, 1985.

\begin{tabular}{|c|c||c|c|c|}
\hline$N$ & $L$ & $e(\omega)$ & $e(\rho)$ & $e\left(f_{4}\right)$ \\
\hline \hline \multicolumn{5}{|c|}{ Algorithm 4.7 } \\
\hline 170 & 84 & $1.4325 \mathrm{e}-11$ & $5.8613 \mathrm{e}-10$ & $6.2146 \mathrm{e}-10$ \\
\hline 170 & 169 & $2.5629 \mathrm{e}-10$ & $3.2084 \mathrm{e}-07$ & $9.7832 \mathrm{e}-12$ \\
\hline 512 & 84 & $4.6367 \mathrm{e}-08$ & $8.3982 \mathrm{e}-06$ & $4.8472 \mathrm{e}-05$ \\
\hline 512 & 511 & $1.1102 \mathrm{e}-14$ & $7.8241 \mathrm{e}-12$ & $3.0388 \mathrm{e}-11$ \\
\hline 1024 & 84 & $1.0560 \mathrm{e}-10$ & $1.7671 \mathrm{e}-07$ & $1.1728 \mathrm{e}-07$ \\
\hline 1024 & 500 & $1.9984 \mathrm{e}-14$ & $2.9797 \mathrm{e}-11$ & $7.8334 \mathrm{e}-11$ \\
\hline 1024 & 1000 & $1.2254 \mathrm{e}-14$ & $1.0122 \mathrm{e}-11$ & $8.5558 \mathrm{e}-11$ \\
\hline \hline \multicolumn{5}{|c|}{ Algorithm 4.8 } \\
\hline 170 & 84 & $7.5935 \mathrm{e}-08$ & $6.3152 \mathrm{e}-06$ & $2.4869 \mathrm{e}-08$ \\
\hline 170 & 169 & $3.1123 \mathrm{e}-11$ & $2.7379 \mathrm{e}-09$ & $7.0488 \mathrm{e}-12$ \\
\hline 512 & 84 & $2.7270 \mathrm{e}-05$ & $1.3895 \mathrm{e}-02$ & $3.1438 \mathrm{e}-02$ \\
\hline 512 & 509 & $2.7420 \mathrm{e}-12$ & $1.0926 \mathrm{e}-09$ & $3.8504 \mathrm{e}-11$ \\
\hline 1024 & 1000 & $7.1054 \mathrm{e}-15$ & $2.5256 \mathrm{e}-09$ & $7.9915 \mathrm{e}-11$ \\
\hline
\end{tabular}

Table 6.5: Results for $f_{4}$ from Example 6.5.

[9] D. Potts, G. Steidl, M. Tasche, Fast Fourier transforms for nonequispaced data: A tutorial, in: J. J. Benedetto, P. J. S. G. Ferreira (Eds.), Modern Sampling Theory: Mathematics and Applications, Birkhäuser, Boston, 2001, pp. $247-270$.

[10] J. Keiner, S. Kunis, D. Potts, NFFT 3.0, C subroutine library, http://www.tu-chemnitz.de/ potts/nfft (2006).

[11] S. Kunis, D. Potts, Stability results for scattered data interpolation by trigonometric polynomials, SIAM J. Sci. Comput. 29 (2007) 1403 - 1419.

[12] A. Björck, Numerical Methods for Least Squares Problems, SIAM, Philadelphia, 1996.

[13] Y. Hua, T. K. Sarkar, Matrix pencil method for estimating parameters of exponentially damped/undamped sinusoids in noise, IEEE Trans. Acoust. Speech Signal Process. 38 (1990) $814-824$.

[14] T. K. Sarkar, O. Pereira, Using the matrix pencil method to estimate the parameters of a sum of complex exponentials, IEEE Antennas and Propagation Magazine 37 (1995) 48 - 55.

[15] R. Roy, T. Kailath, ESPRIT-estimation of signal parameters via rotational invariance techniques, IEEE Trans. Acoustic Speech Signal Process. 37 (1989) $984-994$.

[16] R. Roy, T. Kailath, ESPRIT-estimation of signal parameters via rotational invariance techniques, in: L. Auslander, F.A. Grünbaum, J.W. Helton, T. Kailath, P. Khargoneka, S. Mitter (Eds.), Signal Processing, Part II: Control Theory and Applications, Springer, New York, 1990, pp. 369411.

[17] D. G. Manolakis, V. K. Ingle, S. M. Kogon, Statistical and Adaptive Signal Processing, McGraw-Hill, Boston, 2005.

[18] P. Strobach, Fast recursive low-rank linear prediction frequency estimation algorithms, IEEE Trans. Signal Process. 44 (1996) 834 - 847.

[19] P. Strobach, Total least squares phased averaging and 3-D ESPRIT for joint azimuth-elevation-carrier estimation, IEEE Trans. Signal Process. 59 (2001) $54-62$.

[20] N. J. Higham, Accuracy and Stability of Numerical Algorithms, SIAM, Philadelphia, 1996.

[21] W. B. Jones, O. Njåstad, H. Waadeland, Application of Szegô polynomials to frequency analysis, SIAM J. Math. Anal. 25 (1994) 491 - 512.

[22] H. N. Mhaskar, J. Prestin, On local smoothness classes of periodic functions, J. Fourier Anal. Appl. 11 (2005) 353 - 373. 